\title{
Die Dichtigkeit der Körperbehaarung bei den Chinesen.
}

\author{
Von
}

\section{Toratoshi Taniguchi und Yutaka Kurita.}

Aus dem Anatomischen Institut der Keio Universität, Tokyo.

Inhalt.

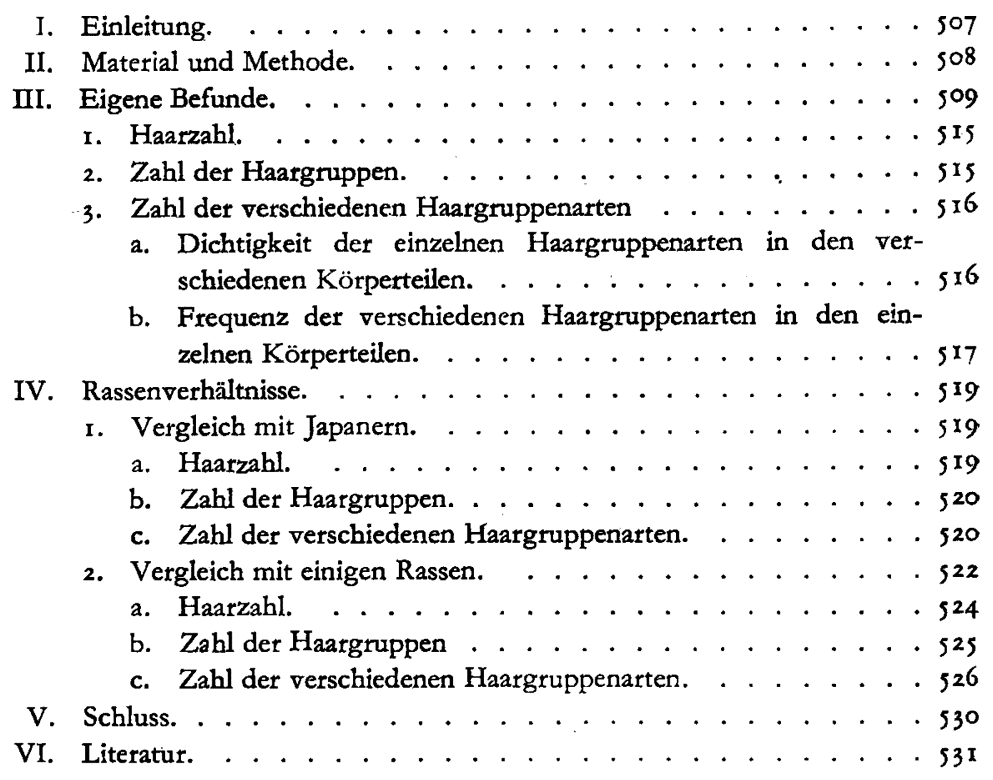

\section{Einleitung.}

Bezüglich der Dichtigkeit der Körperbehaarung bei einigen Menschenrassen beschäftigten sich Taniguchi und $\mathrm{H}$. Shiba y a ma (1935), sie haben daran anschliessend auch über die Dichtigkeit der Körperbehaarung bei den Japanern eingehende Untersuchungen publiziert.

In der vorliegenden Arbeit haben wir die Zahl der Haare, Haargruppen und verschiedenen Haargruppenarten in den verschiedenen Körperteilen bei den Chinesen eingehend studiert und mit denen bei Japanern bzw. einigen Rassen verglichen. 


\section{Material und Methode.}

Als Material wählten wir die Hautstücke von I I erwachsenen Chinesen, wie es die Tabelle I näher zeigt. Die Leichen wurden alle mit Formol-Alkohol-Lösung im ganzen Körper injiziert und dann in 10\% Formollösung konserviert. Die Hautstücke vọn ca. $1.2 \mathrm{qcm}$ Grösse mit genügendem Unterhautgewebe wurden sorgfältig mit dem Skalpell herausgeschnitten, in Zelloidin eingebettet und in Flächenschnittserien von $40 \mu$ Dicke zerlegt. Die Färbung geschah mit Hämatoxylin-(Hansen)-Eosin.

Die folgenden is Körperteile wurden beobachtet.

I. Kopf: I. Scheitel, 2. Stirn.

II. Hals.

III. Rumpf :
4. Brust,
5. Bauch,
6. Rücken,
7. Gesäss.

IV. Obere Extremität :

8. Oberarm (Beugeseite der mittleren Höhe),

9. Oberarm (Streckseite der mittleren Höhe),

ıo. Vorderarm (Beugeseite der mittleren Höhe),

I I. Vorderarm (Streckseite der mittleren Höhe).

V. Untere Extremität :

I 2. Oberschenkel (Medialseite der oberen Höhe),

13. Oberschenkel (Lateralseite der oberen Höhe),

14. Unterschenkel (Beugeseite der mittleren Höhe),

I . Unterschenkel (Streckseite der mittleren Höhe).

Wir kommen hier der angenehmen Pflicht nach, der Peking Universität für die freigebige Überlassung des wertvollen Materials den tiefsten Dank zu sagen.

Tabelle I. Angewendetes Material.

\begin{tabular}{|c|c|c|c|c|c|c|c|}
\hline Fall & $\begin{array}{c}\text { Leichen- } \\
\text { nummer }\end{array}$ & Geschlecht & Alter & Fall & $\begin{array}{c}\text { Leichen- } \\
\text { nummer }\end{array}$ & Geschlecht & Alter \\
\hline I & 15 & $\delta$ & 26 & 7 & 22 & $\delta$ & 28 \\
\hline 2 & 16 & $\delta$ & 25 & 8 & 23 & $\delta$ & 24 \\
\hline 3 & 18 & $\delta$ & 23 & 9 & 6 & $\delta$ & 35 \\
\hline 4 & 19 & $\delta$ & 25 & 10 & 7 & $\delta$ & 26 \\
\hline 5 & 20 & $\delta$ & 27 & 11 & 17 & $\delta$ & 32 \\
\hline 6 & 21 & $\delta$ & 29 & & & & \\
\hline
\end{tabular}




\section{Eigene Befunde.}

Tabelle 2. Haarzahl (in I $\mathrm{qcm}$ ).

\begin{tabular}{|c|c|c|c|c|c|c|c|c|c|c|c|c|}
\hline$\underbrace{\text { Fall }}_{\text {Körperteile }}$ & I & 2 & 3 & 4 & 5 & 6 & 7 & 8 & 9 & I0 & II & $\begin{array}{l}\text { Durch- } \\
\text { schnitt }\end{array}$ \\
\hline Scheitel & 630.0 & 365.0 & $4 \times 2.0$ & 340.0 & 430.0 & 487.5 & 410.0 & - & - & 一 & - & 439 \\
\hline Stirn & $7 \times 2.5$ & 487.0 & 597.5 & 882.5 & 705.0 & 1092.5 & 1025.0 & 1265.0 & - & - & 一 & 846 \\
\hline Hals & 57.5 & 90.0 & $\mathrm{x} 80.0$ & 92.5 & 72.5 & 50.0 & 47.5 & 85.0 & 105.0 & 67.5 & 92.5 & 86 \\
\hline Brust & 65.0 & 62.5 & 135.0 & 97.5 & 52.5 & 60.0 & 67.5 & 55.0 & 55.0 & 75.0 & 55.0 & 71 \\
\hline Bauch & 55.0 & 40.0 & 67.6 & 37.5 & 55.0 & 65.0 & 35.0 & 52.5 & 57.5 & 95.0 & 22.5 & 53 \\
\hline Rücken & 75.0 & 60.0 & 122.5 & 65.0 & 97.5 & 42.5 & - & 95.0 & 100.0 & 85.0 & 82.5 & 83 \\
\hline Gesäss & 75.0 & 97.5 & 92.5 & 70.0 & 60.0 & 67,5 & 62.5 & 77.5 & 65.0 & 105.0 & 87.5 & $7^{8}$ \\
\hline Oberarm (Bs) & 47.5 & 67.5 & 92.5 & 35.0 & 70.0 & 90.0 & 137.5 & 55.0 & - & 57.5 & 30.0 & 69 \\
\hline Oberarm (Ss) & 75.0 & 50.0 & II 7.5 & 95.0 & 95.0 & 62.5 & 67.5 & 105.0 & 5265 & 100.0 & 67.5 & $8 \mathrm{I}$ \\
\hline Vorderarm (Bs) & 47.5 & 47.5 & 62.5 & 30.0 & 35.0 & 97.5 & 57.5 & $47 \cdot 5$ & 65.0 & 55.0 & 60.0 & 55 \\
\hline Vorderarm (Ss) & 107.5 & 112.5 & ros.o & 70.0 & 70.0 & 95.0 & 75.0 & 102.5 & 82.5 & I 12.5 & 52.5 & 90 \\
\hline Obersch. (Ms) & 72.5 & 65.0 & 62.5 & 35.0 & 65.0 & 70.0 & 57.0 & 52.5 & 65.0 & 52.5 & 22.5 & 57 \\
\hline Obersch. (Ls) & 67.5 & 57.5 & 77.5 & 一 & 70.0 & 37.5 & I 10.0 & 60.0 & 62.5 & 75.0 & 97.5 & 72 \\
\hline Untersch. (Bs) & 72.5 & 35.0 & 70.0 & 42.5 & 77.5 & 62.5 & 92.5 & 62.5 & 45.0 & 100.0 & 65.0 & 66 \\
\hline Untersch. (Ss) & 62.5 & 67.5 & 75.0 & 50.0 & 45.0 & 62.5 & 85.0 & 70.0 & 70.0 & 65.0 & 45.0 & 64 \\
\hline
\end{tabular}

- = nicht bestätigt.

Tabelle 3. Haarzahl (in I qcm).

\begin{tabular}{|c|c|c|c|c|c|c|c|c|c|c|c|c|c|}
\hline Körperteile & & I & 2 & 3 & 4 & 5 & 6 & 7 & 8 & 9 & 10 & I I & $\begin{array}{l}\text { Durch- } \\
\text { schnitt }\end{array}$ \\
\hline ganzer Körper & $\begin{array}{l}\text { Kopf } \\
\text { Rumpf } \\
\text { Extremitäten }\end{array}$ & $\begin{array}{r}671 \\
68 \\
69\end{array}$ & $\begin{array}{r}42 \mathrm{I} \\
65 \\
63 \\
\end{array}$ & $\begin{array}{r}505 \\
105 \\
84 \\
\end{array}$ & $\begin{array}{r}611 \\
68 \\
\end{array}$ & $\begin{array}{r}568 \\
67 \\
67 \\
\end{array}$ & $\begin{array}{r}790 \\
59 \\
73\end{array}$ & $\begin{array}{c}7 \times 8 \\
- \\
86\end{array}$ & $\begin{array}{l}7 \\
71 \\
70\end{array}$ & $7 \mathrm{I}$ & $\begin{array}{l}- \\
90 \\
78\end{array}$ & $\begin{array}{l}- \\
63 \\
56\end{array}$ & $\begin{array}{r}612 \\
73 \\
72\end{array}$ \\
\hline Rumpf & $\begin{array}{l}\text { Dorsalseite } \\
\text { Ventralseite }\end{array}$ & $\begin{array}{l}75 \\
60\end{array}$ & $\begin{array}{l}79 \\
51 \\
\end{array}$ & $\begin{array}{l}108 \\
101\end{array}$ & $\begin{array}{l}68 \\
68 \\
\end{array}$ & $\begin{array}{l}79 \\
54 \\
\end{array}$ & $\begin{array}{l}55 \\
63\end{array}$ & $5 \mathrm{I}$ & $\begin{array}{l}87 \\
54 \\
\end{array}$ & $\begin{array}{l}83 \\
57 \\
\end{array}$ & $\begin{array}{l}95 \\
85\end{array}$ & $\begin{array}{l}86 \\
39\end{array}$ & $\begin{array}{l}82 \\
62\end{array}$ \\
\hline $\begin{array}{c}\text { Extremitäten } \\
\text { (a) }\end{array}$ & $\begin{array}{l}\text { obere Extr. } \\
\text { untere Extr. }\end{array}$ & $\begin{array}{l}69 \\
69\end{array}$ & $\begin{array}{l}70 \\
56\end{array}$ & $\begin{array}{l}95 \\
72\end{array}$ & 58 & $\begin{array}{l}68 \\
65 \\
\end{array}$ & $\begin{array}{l}86 \\
59\end{array}$ & $\begin{array}{l}85 \\
87\end{array}$ & $\begin{array}{l}78 \\
62\end{array}$ & $\overline{6 x}$ & $\begin{array}{l}82 \\
74\end{array}$ & $\begin{array}{l}53 \\
59\end{array}$ & $\begin{array}{l}74 \\
66\end{array}$ \\
\hline $\begin{array}{c}\text { Extremitäten } \\
\text { (b) }\end{array}$ & $\begin{array}{l}\text { Proximalis } \\
\text { Distalis }\end{array}$ & $\begin{array}{l}65 \\
74 \\
\end{array}$ & $\begin{array}{l}60 \\
66 \\
\end{array}$ & $\begin{array}{l}88 \\
79 \\
\end{array}$ & 48 & $\begin{array}{l}77 \\
57 \\
\end{array}$ & $\begin{array}{l}65 \\
80 \\
\end{array}$ & $\begin{array}{l}94 \\
78 \\
\end{array}$ & $\begin{array}{l}69 \\
7 x \\
\end{array}$ & $\overline{66}$ & $\begin{array}{l}72 \\
84\end{array}$ & $\begin{array}{l}55 \\
56 \\
\end{array}$ & $\begin{array}{l}72 \\
69\end{array}$ \\
\hline $\begin{array}{c}\text { obere Extr. } \\
\text { (a) }\end{array}$ & $\begin{array}{l}\text { Oberarm } \\
\text { Vorderarm }\end{array}$ & $\begin{array}{l}6 \mathrm{I} \\
78\end{array}$ & $\begin{array}{l}59 \\
80 \\
\end{array}$ & $\begin{array}{r}105 \\
84\end{array}$ & $\begin{array}{l}65 \\
50\end{array}$ & $\begin{array}{l}83 \\
53 \\
\end{array}$ & $\begin{array}{l}76 \\
96\end{array}$ & $\begin{array}{r}103 \\
66\end{array}$ & $\begin{array}{l}80 \\
75\end{array}$ & $\overline{74}$ & $\begin{array}{l}79 \\
84\end{array}$ & $\begin{array}{l}49 \\
57\end{array}$ & $\begin{array}{l}76 \\
72\end{array}$ \\
\hline $\begin{array}{c}\text { obere Extr. } \\
\text { (b) }\end{array}$ & $\begin{array}{l}\text { Streckseite } \\
\text { Beugeseite }\end{array}$ & $\begin{array}{l}91 \\
48 \\
\end{array}$ & $\begin{array}{l}8 \mathrm{I} \\
58 \\
\end{array}$ & $\begin{array}{l}38 \\
56 \\
\end{array}$ & $\begin{array}{l}83 \\
33 \\
\end{array}$ & $\begin{array}{l}83 \\
53 \\
\end{array}$ & $\begin{array}{l}79 \\
94 \\
\end{array}$ & $\begin{array}{l}71 \\
98\end{array}$ & $\begin{array}{r}104 \\
5 \mathrm{r} \\
\end{array}$ & 68 & $\begin{array}{r}106 \\
57 \\
\end{array}$ & $\begin{array}{l}6 \mathrm{I} \\
45 \\
\end{array}$ & $\begin{array}{l}79 \\
59 \\
\end{array}$ \\
\hline untere Extr. & $\begin{array}{l}\text { Oberschenkel } \\
\text { Unterschenkel }\end{array}$ & $\begin{array}{l}70 \\
68\end{array}$ & $\begin{array}{l}61 \\
51\end{array}$ & $\begin{array}{l}7 \mathrm{x} \\
73\end{array}$ & - & $\begin{array}{l}68 \\
6 \mathrm{r}\end{array}$ & $\begin{array}{l}54 \\
63\end{array}$ & $\begin{array}{l}84 \\
89\end{array}$ & $\begin{array}{l}57 \\
67\end{array}$ & $\begin{array}{l}64 \\
58\end{array}$ & $\begin{array}{l}64 \\
83\end{array}$ & $\begin{array}{l}62 \\
55\end{array}$ & $\begin{array}{l}66 \\
75\end{array}$ \\
\hline
\end{tabular}


Tabelle 4, Haargruppenzahl (in I qcm).

\begin{tabular}{|c|c|c|c|c|c|c|c|c|c|c|c|c|}
\hline$\underbrace{\text { Fall }}_{\text {Körperteile }}$ & I & 2 & 3 & 4 & 5 & 6 & 7 & 8 & 9 & Io & II & $\begin{array}{l}\text { Durch- } \\
\text { schnitt }\end{array}$ \\
\hline Scheitel & $x 87.5$ & I 15.0 & I52.0 & 132.5 & 152.5 & 145.0 & 155.0 & - & - & - & - & 150 \\
\hline Hals & 42.5 & 47.5 & 69.0 & 47.5 & 27.5 & 37.5 & 37.5 & 35.0 & 40.0 & 47.5 & 52.5 & 45 \\
\hline Brust & 47.5 & 45.0 & 72.5 & 65.0 & 42.5 & 37.5 & 37.5 & 35.0 & 32.5 & 45.0 & 35.0 & 45 \\
\hline Bauch & 35.0 & 27.5 & 40.0 & 30.0 & 32.5 & 45.0 & 30.0 & 35.0 & 37.5 & 50.0 & 22.5 & 35 \\
\hline Rücken & 37.5 & 50.0 & 65.0 & 45.0 & 47.) & 32.5 & - & 45.0 & so.o & 52.5 & 62.5 & 49 \\
\hline Gesäss & 47.5 & 60.0 & 57.5 & 37.5 & 37.5 & 47.5 & 40.0 & 50.0 & 35.0 & 70.0 & 62.5 & 50 \\
\hline Oberarm (Bs) & 32.5 & 55.0 & 80.0 & 25.0 & 47.5 & 72.5 & I32.5 & 37.5 & - & 37.5 & 30.0 & 56 \\
\hline Oberarm (Ss) & 45.0 & 37.5 & 82.5 & 57.5 & 50.0 & 45.0 & 42.5 & 52.5 & 30.0 & 57.5 & 55.0 & 51 \\
\hline Vorderarm (Bs) & 47.5 & 40.0 & 52.5 & 30.0 & 27.5 & 92.5 & 57.5 & 42.5 & 37.5 & 50,0 & 57.5 & 50 \\
\hline Vorderarm (Ss) & 65.0 & 55.0 & 60.0 & 37.5 & 42.5 & 47.5 & 6o.o & 55.0 & 40.0 & 62.5 & 50.0 & 53 \\
\hline Obersch. (Ms) & 52.5 & 35.0 & 35.0 & 22.5 & 47.5 & 42.5 & 40.0 & 25.0 & 35.0 & 32.5 & 22.5 & 36 \\
\hline Obersch. (Ls) & 55.0 & 25.0 & 47.5 & - & 42.5 & 35.0 & 102.5 & 37.5 & 35.0 & 52.5 & 65.0 & 49 \\
\hline Untersch. (Bs) & 50.0 & 25.0 & 42.5 & 37.5 & 65.0 & 40.0 & 85.0 & 35.0 & 32.5 & 62.5 & 45.0 & 47 \\
\hline Untersch. (Ss) & 50.0 & 50.0 & 70.0 & 27.5 & 32.5 & 45.0 & 85.0 & 45.0 & $55.0^{\circ}$ & 42.5 & 27.5 & $4^{8}$ \\
\hline
\end{tabular}

Tabelle s. Haargruppenzahl (in I qcm).

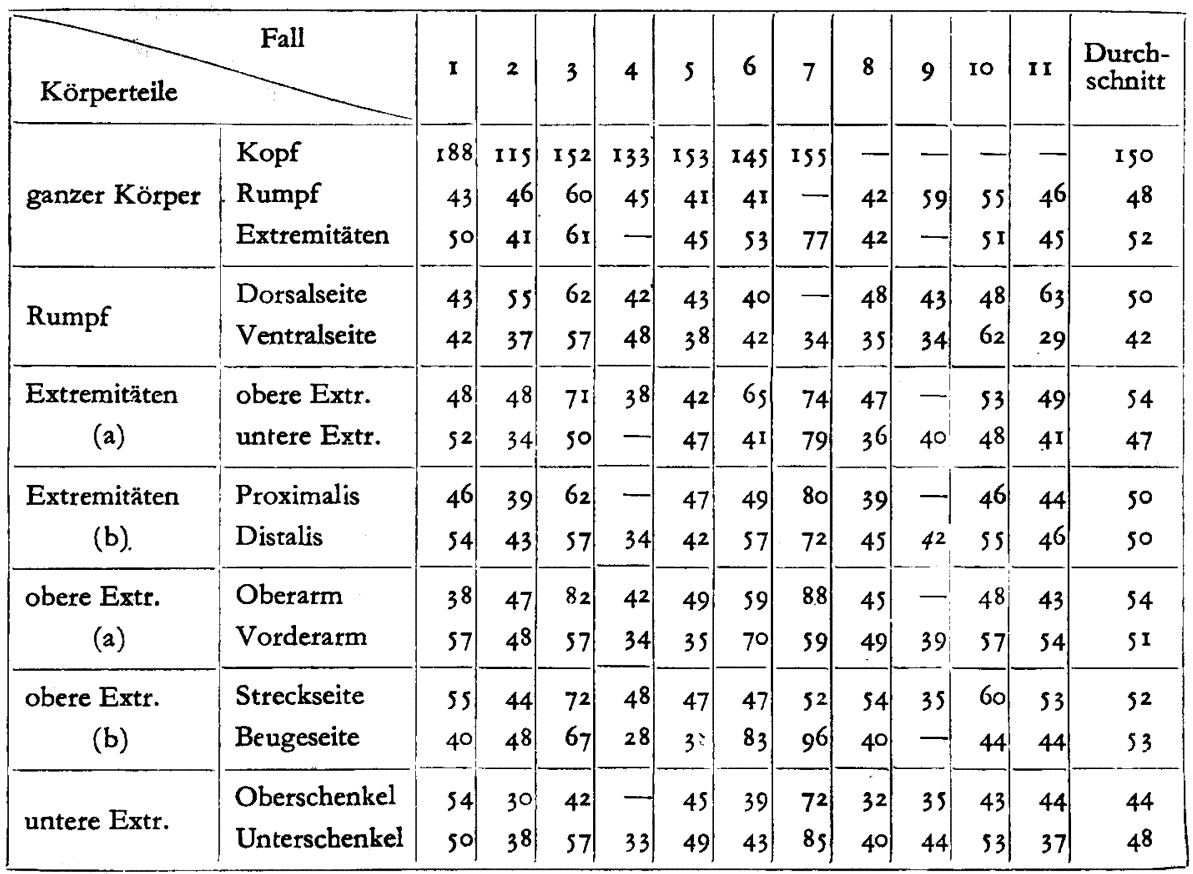


Die Dichtigkeit der Körperbehaarung bei den Chinesen.

Tabelle 6. I-haarige Gruppe (I $\mathrm{qcm}$ ).

\begin{tabular}{|c|c|c|c|c|c|c|c|c|c|c|c|c|c|}
\hline \multicolumn{2}{|c|}{$\begin{array}{ll}\text { Körperteile } & \text { Fall } \\
\end{array}$} & I & 2 & 3 & 4 & 5 & 6 & 7 & 8 & 9 & 10 & II & $\begin{array}{l}\text { Durch- } \\
\text { schnitt }\end{array}$ \\
\hline \multirow{3}{*}{ ganzer Körper } & Kopf & 13 & Io & IS & 15 & o) & 10 & IS & $\longrightarrow$ & 一 & $\cdots$ & - & I I \\
\hline & Rumpf & 68 & 29 & 23 & 24 & 18 & 18 & $\longrightarrow$ & 16 & Io & 24 & 57 & 21 \\
\hline & Extremitäten & 34 & $2 \mathrm{I}$ & 38 & - & 27 & 38 & 67 & I9! & $\cdots$ & 26 & 56 & 32 \\
\hline \multirow{2}{*}{ Rumpf } & Dorsalseite & 75 & 35 & 22 & 19 & 14 & IS & - & 14 & 5 & 32 & 85 & 21 \\
\hline & Ventralseite & 60 & 22 & 24 & 28 & 22 & 20 & 18 & 17 & 14 & IS & 29 & 20 \\
\hline \multirow{2}{*}{$\begin{array}{c}\text { Extremitäten } \\
\text { (a) }\end{array}$} & obere Extr. & 32 & 28 & 47 & 19 & 23 & 47 & 63 & 20 & - & 26 & 53 & 34 \\
\hline & untere Extr. & 36 & 13 & 29 & - & $3 r$ & 29 & $7 \mathrm{I}$ & 18 & $2 \mathrm{I}$ & 26 & 58 & 29 \\
\hline \multirow{2}{*}{$\begin{array}{c}\text { Extremitäten } \\
\text { (b) }\end{array}$} & Proximalis & 29 & I9 & $4 \mathrm{r}$ & -1 & 24 & 37 & 67 & IS & 一 & 22 & 55 & 30 \\
\hline & Distalis & 38 & 22 & 35 & I9 & 30 & 39 & 67 & 23 & 19 & $3 I$ & 59 & 33 \\
\hline \multirow{2}{*}{$\begin{array}{c}\text { obere Extr. } \\
\text { (a) }\end{array}$} & Oberarm & 20 & 30 & 64 & I9 & 23 & 44 & 74. & I7 & - & 18 & 49 & 34 \\
\hline & Vorderarm & 43 & 25 & 30 & I8 & 23 & 50 & 52 & 23 & 7. & 34 & 57 & 33 \\
\hline \multirow{2}{*}{$\begin{array}{c}\text { obere Extr. } \\
\text { (b) }\end{array}$} & Streckseite & 30 & 18 & 38 & 14 & 22 & 22 & 33 & 10 & $\rightarrow$ & I9 & 60 & 24 \\
\hline & Beugeseite & 33 & 38 & 57 & 23 & 24 & 72 & 93 & 29 & 5 & 33 & 45 & 43 \\
\hline \multirow{2}{*}{ untere Extr. } & Oberschenkel & 38 & 7 & 17 & - & 24 & 30 & 59 & 12 & 12 & 25 & 60 & 25 \\
\hline & Unterschenkel & 33 & 18 & 40 & x9 & 37 & 27 & 82 & 23 & 30 & 27 & 55 & 32 \\
\hline
\end{tabular}

Tabelle 7. 2-haarige Haargruppe (I $\mathrm{qcm}$ ).

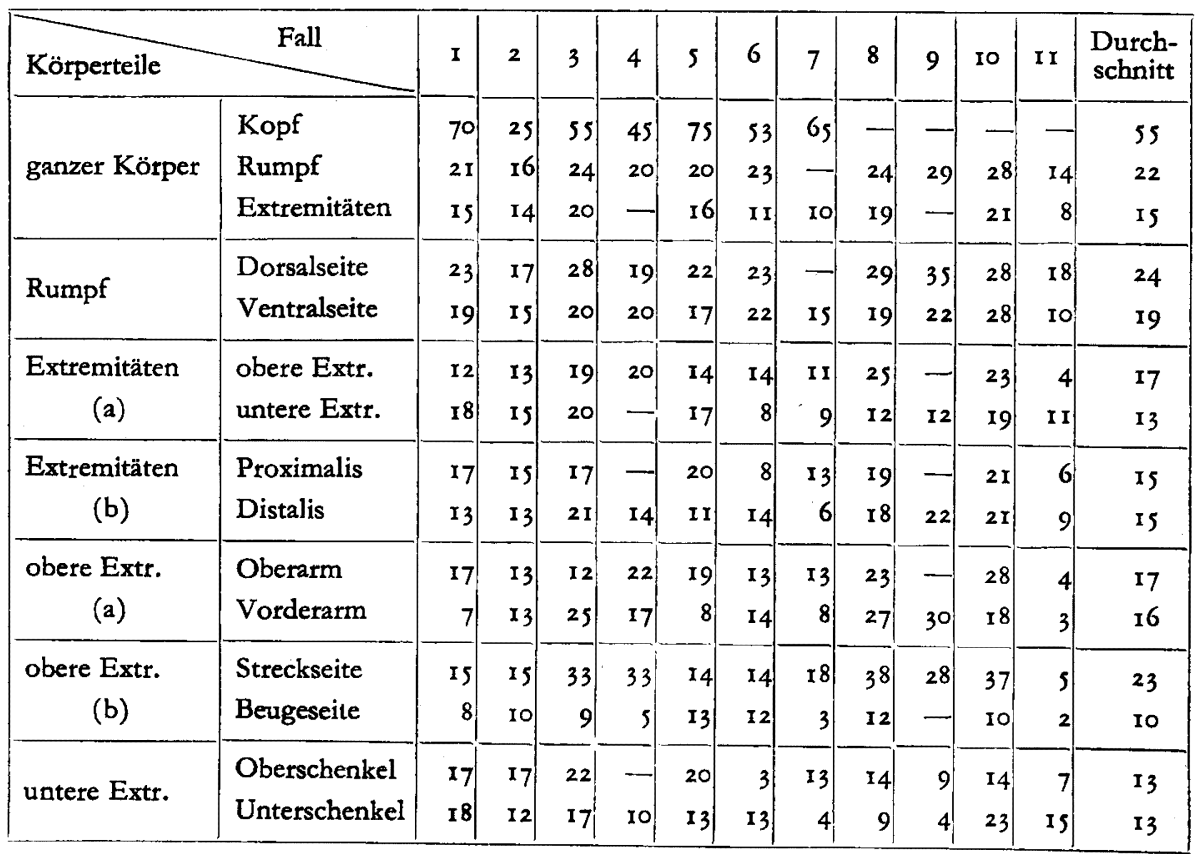


Tabelle 8. 3-haarige Gruppe.

\begin{tabular}{|c|c|c|c|c|c|c|c|c|c|c|c|c|c|}
\hline \multicolumn{2}{|l|}{ Körperteile } & $\mathbf{I}$ & 2 & 3 & 4 & 5 & 6 & 7 & 8 & 9 & Io & II & $\begin{array}{l}\text { Durch- } \\
\text { schnitt }\end{array}$ \\
\hline ganzer Körper & $\begin{array}{l}\text { Kopf } \\
\text { Rumpf } \\
\text { Extremitäten }\end{array}$ & $\begin{array}{r}25 \\
3 \\
3\end{array}$ & $\begin{array}{r}30 \\
2 \\
7\end{array}$ & $\begin{array}{r}60 \\
13 \\
4\end{array}$ & $\begin{array}{r}63 \\
2 \\
1\end{array}$ & $\begin{array}{r}40 \\
4 \\
4\end{array}$ & \begin{tabular}{r|}
23 \\
2 \\
6
\end{tabular} & $\frac{43}{-}$ & -1 & $\begin{array}{r}- \\
2 \\
3\end{array}$ & 5 & $\begin{array}{r}- \\
2 \\
4\end{array}$ & $\begin{array}{r}41 \\
4 \\
4\end{array}$ \\
\hline Rumpf & $\begin{array}{l}\text { Dorsalseite } \\
\text { Ventralseite }\end{array}$ & 5 & 4 & $\begin{array}{l}12 \\
\mathrm{I}_{3}\end{array}$ & $\begin{array}{l}4 \\
0\end{array}$ & 8 & $\begin{array}{l}3 \\
0\end{array}$ & -4 & 5 & 3 & $\begin{array}{l}4 \\
5\end{array}$ & $\begin{array}{l}3 \\
0\end{array}$ & $\begin{array}{l}5 \\
2\end{array}$ \\
\hline $\begin{array}{l}\text { Extremitäten } \\
\text { (a) }\end{array}$ & $\begin{array}{l}\text { obere Extr. } \\
\text { untere Extr. }\end{array}$ & 5 & $\begin{array}{l}7 \\
6\end{array}$ & $\begin{array}{l}5 \\
2\end{array}$ & - & 7 & 5 & $\begin{array}{l}\mathbf{1} \\
0\end{array}$ & $\begin{array}{l}4 \\
8\end{array}$ & -1 & $\begin{array}{l}4 \\
4\end{array}$ & $\mathrm{r}$ & $\begin{array}{l}4 \\
4\end{array}$ \\
\hline $\begin{array}{l}\text { Extremitäten } \\
\text { (b) }\end{array}$ & $\begin{array}{l}\text { Proximalis } \\
\text { Distalis }\end{array}$ & 4 & $\begin{array}{l}6 \\
7\end{array}$ & 6 & - & 5 & 5 & I & t) & - & 3 & 5 & $\begin{array}{l}4 \\
4\end{array}$ \\
\hline $\begin{array}{l}\text { obere Extr. } \\
\text { (a) }\end{array}$ & $\begin{array}{l}\text { Oberarm } \\
\text { Vorderarm }\end{array}$ & $\begin{array}{l}2 \\
8\end{array}$ & $\begin{array}{r}4 \\
10\end{array}$ & $\begin{array}{l}7 \\
2\end{array}$ & $\begin{array}{l}2 \\
0\end{array}$ & 8 & $\begin{array}{l}3 \\
7\end{array}$ & 2 & 7 & $\begin{array}{r}-- \\
3\end{array}$ & $\begin{array}{l}2 \\
5\end{array}$ & 2 & $\begin{array}{l}3 \\
4\end{array}$ \\
\hline $\begin{array}{l}\text { obere Extr. } \\
\text { (b) }\end{array}$ & $\begin{array}{l}\text { Streckseite } \\
\text { Beugeseite }\end{array}$ & 9 & 14 & $\begin{array}{l}7 \\
2\end{array}$ & 2 & $\begin{array}{r}12 \\
2\end{array}$ & $\begin{array}{l}9 \\
0\end{array}$ & 2 & 7 & 3 & $s$ & 2 & $\begin{array}{l}6 \\
1\end{array}$ \\
\hline Untere Extr. & $\begin{array}{l}\text { Oberschenkel } \\
\text { Unterschenkel }\end{array}$ & $\begin{array}{l}0 \\
0\end{array}$ & $\begin{array}{l}8 \\
4\end{array}$ & 4 & -1 & 2 & 7 & o & 7 & Io & 4 & $\begin{array}{l}7 \\
4\end{array}$ & $\begin{array}{l}5 \\
3\end{array}$ \\
\hline
\end{tabular}

Tabelle 9. Mehr als 3-haarige Haargruppe.

\begin{tabular}{|c|c|c|c|c|c|c|c|c|c|c|c|c|c|}
\hline \multicolumn{2}{|c|}{$\begin{array}{ll}\text { Körperteile } & \text { Fall } \\
\end{array}$} & $\mathbf{I}$ & 2 & 3 & 4 & 5 & 6 & 7 & 8 & 9 & 10 & II & $\begin{array}{l}\text { Durch- } \\
\text { schnitt }\end{array}$ \\
\hline ganzer Körper & $\begin{array}{l}\text { Kopf } \\
\text { Rumpf } \\
\text { Extremitäten }\end{array}$ & $\begin{array}{r}8 \mathrm{I} \\
0 \\
\mathrm{I}\end{array}$ & $\begin{array}{r}50 \\
0 \\
0\end{array}$ & $\begin{array}{r}25 \\
1 \\
0\end{array}$ & $\begin{array}{r}10 \\
0 \\
0\end{array}$ & $\begin{array}{r}38 \\
0 \\
0\end{array}$ & 6o & 33 & -1 & - & - & - & $\begin{array}{c}42 \\
0+ \\
0+\end{array}$ \\
\hline Rumpf & $\begin{array}{l}\text { Dorsalseite } \\
\text { Ventralseite }\end{array}$ & $\circ$ & 0 & 2 & o & o & o & -1 & 0 & o & 0 & $\circ$ & $\begin{array}{l}0^{+} \\
0\end{array}$ \\
\hline $\begin{array}{l}\text { Extremitäten } \\
\text { (a) }\end{array}$ & $\begin{array}{l}\text { obere Extr. } \\
\text { untere Extr. }\end{array}$ & 1 & $\circ$ & o & o & $\circ$ & o & 0 & o & - & o & o & $\begin{array}{l}\mathrm{o}^{+} \\
\mathrm{o}^{+}\end{array}$ \\
\hline $\begin{array}{l}\text { Extremitäten } \\
\text { (b) }\end{array}$ & $\begin{array}{l}\text { Proximalis } \\
\text { Distalis }\end{array}$ & I & o & 0 & -1 & o & o & 0 & 0 & I & 0 & 0 & $\begin{array}{l}0^{+} \\
\circ\end{array}$ \\
\hline $\begin{array}{l}\text { obere Extr. } \\
\text { (a) }\end{array}$ & $\begin{array}{l}\text { Oberarm } \\
\text { Vorderarm }\end{array}$ & 2 & o & o & 0 & 0 & of & o & 0 & - & 0 & 0 & $\begin{array}{l}0^{+} \\
\circ\end{array}$ \\
\hline $\begin{array}{l}\text { obere Extr. } \\
\text { (b) }\end{array}$ & $\begin{array}{l}\text { Streckseite } \\
\text { Beugeseite }\end{array}$ & $\begin{array}{l}2 \\
0\end{array}$ & o & 0 & 0 & o & o & o & $\begin{array}{l}\text { of } \\
0\end{array}$ & 0 & $\circ$ & 0 & $\begin{array}{l}0^{+} \\
0\end{array}$ \\
\hline untere Extr. & $\begin{array}{l}\text { Oberschenkel } \\
\text { Unterschenkel }\end{array}$ & $\begin{array}{l}0 \\
0 \\
0\end{array}$ & 0 & : & - & o & o & o & o & 2 & 0 & 0 & $\begin{array}{l}0^{+} \\
0\end{array}$ \\
\hline
\end{tabular}

$\mathrm{o}^{+}=$unter $1 \%$. 
Tabelle ıо. Mittelwert von I I Chinesen in einzelnen Körperteilen.

\begin{tabular}{|c|c|c|c|c|c|c|c|c|c|c|}
\hline \multirow{3}{*}{ Körperteile } & \multicolumn{10}{|c|}{ Zahl in $1 \mathrm{qcm}$ Hautstück } \\
\hline & \multirow{2}{*}{$\begin{array}{c}\text { Haar- } \\
\text { zahl }\end{array}$} & \multicolumn{9}{|c|}{ Haargruppen } \\
\hline & & $\mathrm{I}-\mathrm{Hg}$ & 2-Hg & $3-\mathrm{Hg}$ & $4-\mathrm{Hg}$ & $5-\mathrm{Hg}$ & $6-\mathrm{Hg}$ & $7-\mathrm{Hg}$ & 9-Hg & Summe \\
\hline $\begin{array}{l}\text { Scheitel } \\
\text { Stirn } \\
\text { Hals }\end{array}$ & $\begin{array}{r}439 \\
846 \\
86\end{array}$ & $\frac{11}{17}$ & $\frac{55}{15}$ & $\frac{4 I}{I I}$ & $\frac{22}{2}$ & 14 & 6 & $\stackrel{0+}{-}$ & $\stackrel{0}{+}^{+}$ & $\frac{150}{45}$ \\
\hline $\begin{array}{l}\text { Brust } \\
\text { Bauch } \\
\text { Rücken } \\
\text { Gesäss }\end{array}$ & $\begin{array}{l}71 \\
53 \\
83 \\
78\end{array}$ & $\begin{array}{l}22 \\
18 \\
19 \\
23\end{array}$ & $\begin{array}{l}21 \\
16 \\
24 \\
23\end{array}$ & $\begin{array}{l}3 \\
1 \\
6 \\
4\end{array}$ & $0^{+}$ & & & & & $\begin{array}{l}45 \\
35 \\
49 \\
50\end{array}$ \\
\hline $\begin{array}{l}\text { Oberarm (Bs) } \\
\text { Oberarm (Ss) } \\
\text { Vorderarm (Bs) } \\
\text { Vorderarm (Ss) }\end{array}$ & $\begin{array}{l}69 \\
81 \\
55 \\
60\end{array}$ & $\begin{array}{l}43 \\
25 \\
43 \\
22\end{array}$ & $\begin{array}{r}12 \\
21 \\
7 \\
24\end{array}$ & $\begin{array}{l}1 \\
5 \\
0+ \\
7\end{array}$ & $0^{+}$ & & & & & $\begin{array}{l}56 \\
51 \\
50 \\
53\end{array}$ \\
\hline $\begin{array}{l}\text { Obersch. (Ms) } \\
\text { Obersch. (Ls) } \\
\text { Untersch. (Bs) } \\
\text { Untersch. (Ss) }\end{array}$ & $\begin{array}{l}57 \\
72 \\
66 \\
64\end{array}$ & $\begin{array}{l}18 \\
32 \\
31 \\
33\end{array}$ & $\begin{array}{l}13 \\
12 \\
14 \\
12\end{array}$ & $\begin{array}{l}5 \\
5 \\
2 \\
3\end{array}$ & o+ & & & & & $\begin{array}{l}36 \\
47 \\
47 \\
48\end{array}$ \\
\hline
\end{tabular}

Tabelle I I. Mittelwert von I I Chinesen in allerlei Hauptkörperabschnitten.

\begin{tabular}{|c|c|c|c|c|c|c|c|c|c|c|c|}
\hline \multirow{3}{*}{\multicolumn{2}{|c|}{ Körperteile }} & \multicolumn{10}{|c|}{ Zahl in $1 \mathrm{qcm}$ Hautstück } \\
\hline & & \multirow{2}{*}{$\begin{array}{c}\text { Haar- } \\
\text { zahl }\end{array}$} & \multicolumn{9}{|c|}{ Haargruppen } \\
\hline & & & $\mathrm{I}-\mathrm{Hg}$ & $2-\mathrm{Hg}$ & $3-\mathrm{Hg}$ & $4-\mathrm{Hg}$ & $5-\mathrm{Hg}$ & 6-Hg & $7-\mathrm{Hg}$ & $9-\mathrm{Hg}$ & Summe \\
\hline ganzer Körper & $\begin{array}{l}\text { Kopf* } \\
\text { Rumpf } \\
\text { Extremitäten }\end{array}$ & $\begin{array}{r}612 \\
73 \\
72\end{array}$ & $\begin{array}{l}\text { II } \\
2 \text { I } \\
32\end{array}$ & $\begin{array}{l}55 \\
22 \\
15\end{array}$ & $\begin{array}{r}4 \mathrm{I} \\
4 \\
4\end{array}$ & $\begin{array}{c}22 \\
\text { o+ } \\
\text { ot }\end{array}$ & $\begin{array}{r}14 \\
0 \\
0\end{array}$ & $\begin{array}{l}6 \\
0 \\
0\end{array}$ & $\begin{array}{l}0^{+} \\
0 \\
0\end{array}$ & $\begin{array}{l}0+ \\
0 \\
0\end{array}$ & $\begin{array}{r}r 50 \\
48 \\
52\end{array}$ \\
\hline Rumpf & $\begin{array}{l}\text { Dorsalseite } \\
\text { Ventralseite }\end{array}$ & $\begin{array}{l}82 \\
62\end{array}$ & $\begin{array}{l}2 \mathrm{I} \\
20\end{array}$ & $\begin{array}{l}24 \\
19\end{array}$ & $\begin{array}{l}5 \\
2\end{array}$ & $\begin{array}{l}\mathrm{o}^{+} \\
0\end{array}$ & $\begin{array}{l}0 \\
0\end{array}$ & $\begin{array}{l}\circ \\
\circ\end{array}$ & $\begin{array}{l}\circ \\
\circ\end{array}$ & $\begin{array}{l}\circ \\
\circ\end{array}$ & $\begin{array}{l}50 \\
42\end{array}$ \\
\hline $\begin{array}{l}\text { Extremitäten } \\
\text { (a) }\end{array}$ & $\begin{array}{l}\text { obere Extr. } \\
\text { untere Extr. }\end{array}$ & $\begin{array}{l}74 \\
66\end{array}$ & $\begin{array}{l}34 \\
29\end{array}$ & $\begin{array}{l}17 \\
13\end{array}$ & $\begin{array}{l}4 \\
4\end{array}$ & $\begin{array}{l}\text { ot }^{+} \\
\text {of }^{+}\end{array}$ & $\begin{array}{l}\circ \\
0\end{array}$ & $\begin{array}{l}\circ \\
\circ\end{array}$ & $\begin{array}{l}\circ \\
\circ\end{array}$ & $\begin{array}{l}\circ \\
\circ\end{array}$ & $\begin{array}{l}54 \\
47\end{array}$ \\
\hline $\begin{array}{l}\text { Extremitäten } \\
\text { (b) }\end{array}$ & $\begin{array}{l}\text { Proximalis } \\
\text { Distalis }\end{array}$ & $\begin{array}{l}72 \\
69\end{array}$ & $\begin{array}{l}30 \\
33\end{array}$ & $\begin{array}{l}15 \\
15\end{array}$ & $\begin{array}{l}4 \\
4\end{array}$ & $\begin{array}{l}0^{+} \\
0^{+}\end{array}$ & $\begin{array}{l}\circ \\
0\end{array}$ & $\begin{array}{l}\circ \\
\circ\end{array}$ & $\begin{array}{l}\circ \\
\circ\end{array}$ & $\begin{array}{l}\circ \\
\circ\end{array}$ & $\begin{array}{l}50 \\
50\end{array}$ \\
\hline $\begin{array}{l}\text { obere Extr. } \\
\text { (a) }\end{array}$ & $\begin{array}{l}\text { Oberarm } \\
\text { Vorderarm }\end{array}$ & $\begin{array}{l}7^{6} \\
7^{2}\end{array}$ & $\begin{array}{l}34 \\
33\end{array}$ & $\begin{array}{l}17 \\
16\end{array}$ & $\begin{array}{l}3 \\
4\end{array}$ & $\begin{array}{l}0^{+} \\
0\end{array}$ & $\begin{array}{l}\circ \\
\circ\end{array}$ & $\begin{array}{l}\circ \\
\circ\end{array}$ & $\begin{array}{l}\circ \\
\circ\end{array}$ & $\begin{array}{l}0 \\
0\end{array}$ & $\begin{array}{l}54 \\
51\end{array}$ \\
\hline $\begin{array}{l}\text { obere Extr. } \\
\text { (b) }\end{array}$ & $\begin{array}{l}\text { Streckseite } \\
\text { Beugeseite }\end{array}$ & $\begin{array}{l}79 \\
59\end{array}$ & $\begin{array}{l}24 \\
43\end{array}$ & $\begin{array}{l}23 \\
10\end{array}$ & $\begin{array}{l}6 \\
x\end{array}$ & $\begin{array}{l}0+ \\
0\end{array}$ & $\begin{array}{l}0 \\
0\end{array}$ & $\begin{array}{l}\circ \\
\circ\end{array}$ & $\begin{array}{l}\circ \\
0\end{array}$ & $\begin{array}{l}0 \\
0\end{array}$ & $\begin{array}{l}52 \\
53\end{array}$ \\
\hline untere Extr. & $\begin{array}{l}\text { Oberschenkel } \\
\text { Unterschenkel }\end{array}$ & $\begin{array}{l}66 \\
75\end{array}$ & $\begin{array}{l}25 \\
32\end{array}$ & $\begin{array}{l}13 \\
13\end{array}$ & $\begin{array}{l}5 \\
3\end{array}$ & $\begin{array}{l}\text { o+ } \\
0\end{array}$ & $\begin{array}{l}\circ \\
\circ\end{array}$ & $\begin{array}{l}\circ \\
\circ\end{array}$ & $\begin{array}{l}\circ \\
\circ\end{array}$ & $\begin{array}{l}\circ \\
\circ\end{array}$ & $\begin{array}{l}44 \\
48\end{array}$ \\
\hline
\end{tabular}

* : Haarzahl = $1 / 2$ (Zahl am Scheitel + Zahl an d. Stirn $)$, Haargruppenzahl =dieselbe an d. Scheitel. 
Tabelle I 2. Durchschnittlicher Prozentsatz von I I Chinesen in einzelnen Körperteilen.

\begin{tabular}{|c|c|c|c|c|c|c|c|c|}
\hline \multirow{2}{*}{ Körperteile } & \multicolumn{8}{|c|}{ Haargruppen } \\
\hline & $\mathrm{I}-\mathrm{Hg}$ & $2-\mathrm{Hg}$ & $3-\mathrm{Hg}$ & 4- $\mathrm{Hg}$ & $5-\mathrm{Hg}$ & $6-\mathrm{Hg}$ & $7-\mathrm{Hg}$ & 9- $\mathrm{Hg}$ \\
\hline Scheitel & $7.4 \%$ & $36.9 \%$ & $27.5 \%$ & $14.8 \%$ & $9.3 \%$ & $4.1 \%$ & $\mathrm{o}^{+}$ & $0^{+}$ \\
\hline Hals & 37.8 & 33.3 & $24 \cdot 4$ & 4.4 & & & & \\
\hline Brust & 48.9 & 46.7 & 6.7 & & & & & \\
\hline Bauch & $5 \mathrm{I} .4$ & 45.7 & 2.9 & & & & & \\
\hline Rücken & 38.8 & 49.0 & I 2.2 & $\mathrm{o}^{+}$ & & & & \\
\hline Gesäss & 46.0 & 46.0 & 8.0 & & & & & \\
\hline Oberarm (Bs) & $7^{6.8}$ & 21.4 & I. 8 & & & & & \\
\hline Oberarm (Ss) & 49.0 & 41.2 & 9.8 & o+ & & & & \\
\hline Vorderarm (Bs) & 86.0 & I 3.7 & o+ & & & & & \\
\hline Vorderarm (Ss) & $4 \mathrm{I} .5$ & $45 \cdot 3$ & 13.2 & & & & & \\
\hline Obersch. (Ms) & 50.0 & 36.1 & 13.9 & & & & & \\
\hline Obersch. (Ls) & 65.3 & 24.5 & 10.2 & $0^{+}$ & & & & \\
\hline Untersch. (Bs) & 66.0 & 29.8 & 4.2 & & & & & \\
\hline Untersch. (Ss) & 68.8 & 25.0 & 6.2 & & & & & \\
\hline
\end{tabular}

Tabelle I3. Durchschnittlicher Prozentsatz von I Chinesen in allerlei Hauptkörperabschnitten.

\begin{tabular}{|c|c|c|c|c|c|c|c|c|c|}
\hline \multirow{2}{*}{\multicolumn{2}{|c|}{ Körperteile }} & \multicolumn{8}{|c|}{ Haargruppen } \\
\hline & & \multirow{2}{*}{$\begin{array}{l}\mathrm{I}-\mathrm{Hg} \\
\begin{array}{l}7.3 \% \\
43.8 \\
61.5\end{array}\end{array}$} & \multirow{2}{*}{$\begin{array}{l}2-\mathrm{Hg} \\
37.0 \% \\
45.8 \\
28.9\end{array}$} & \multirow{2}{*}{\begin{tabular}{|c|}
$3-\mathrm{Hg}$ \\
$27.3 \%$ \\
8.3 \\
7.7
\end{tabular}} & \multirow{2}{*}{$\begin{array}{c}4-\mathrm{Hg} \\
15.0 \% \\
0^{+} \\
0^{+}\end{array}$} & \multirow{2}{*}{$\begin{array}{c}5-\mathrm{Hg} \\
9 \cdot 3 \% \\
0 \\
0\end{array}$} & \multirow{2}{*}{$\begin{array}{l}6-\mathrm{Hg} \\
\begin{array}{l}4.0 \% \\
0 \\
0\end{array}\end{array}$} & \multirow{2}{*}{ 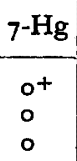 } & \multirow{2}{*}{ 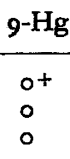 } \\
\hline Ganzer Körper & $\begin{array}{l}\text { Kopf } \\
\text { Rumpf } \\
\text { Extremitäten }\end{array}$ & & & & & & & & \\
\hline Rumpf & $\begin{array}{l}\text { Dorsalseite } \\
\text { Ventralseite }\end{array}$ & $\begin{array}{l}42.0 \\
47.6\end{array}$ & $\begin{array}{l}48.0 \\
45.2\end{array}$ & $\begin{array}{r}10.0 \\
4.8\end{array}$ & $\begin{array}{l}0^{+} \\
0\end{array}$ & $\begin{array}{l}\circ \\
\circ\end{array}$ & $\begin{array}{l}\circ \\
0\end{array}$ & $\begin{array}{l}\circ \\
\circ\end{array}$ & $\begin{array}{l}\circ \\
\circ\end{array}$ \\
\hline Extremitäten (a) & $\begin{array}{l}\text { obere Extr. } \\
\text { untere Extr. }\end{array}$ & $\begin{array}{l}62.9 \\
6 r .7\end{array}$ & $\begin{array}{l}31.5 \\
27.7\end{array}$ & $\begin{array}{l}7.4 \\
8.6\end{array}$ & $\begin{array}{l}0^{+} \\
0^{+}\end{array}$ & $\begin{array}{l}\circ \\
\circ\end{array}$ & $\begin{array}{l}\circ \\
\circ\end{array}$ & $\begin{array}{l}\circ \\
\circ\end{array}$ & $\begin{array}{l}0 \\
0\end{array}$ \\
\hline Extremitäten (b) & $\begin{array}{l}\text { Proximalis } \\
\text { Distalis }\end{array}$ & $\begin{array}{l}60.0 \\
66.0\end{array}$ & $\begin{array}{l}30.0 \\
30.0\end{array}$ & $\begin{array}{l}8.0 \\
8.0\end{array}$ & $\begin{array}{l}0^{+} \\
0\end{array}$ & $\begin{array}{l}\circ \\
\circ\end{array}$ & $\begin{array}{l}0 \\
0\end{array}$ & $\begin{array}{l}0 \\
0\end{array}$ & $\begin{array}{l}0 \\
0\end{array}$ \\
\hline obere Extr. (a) & $\begin{array}{l}\text { Oberarm } \\
\text { Vorderarm }\end{array}$ & $\begin{array}{l}62.9 \\
64.7\end{array}$ & $\begin{array}{l}3 I .5 \\
3 I .4\end{array}$ & $\begin{array}{l}5.6 \\
7.8\end{array}$ & $\begin{array}{l}0+ \\
0\end{array}$ & $\begin{array}{l}\circ \\
0\end{array}$ & $\begin{array}{l}0 \\
\circ\end{array}$ & $\begin{array}{l}\circ \\
\circ\end{array}$ & $\begin{array}{l}\circ \\
\circ\end{array}$ \\
\hline obere Extr. (b) & $\begin{array}{l}\text { Streckseite } \\
\text { Beugeseite }\end{array}$ & $\begin{array}{l}46.1 \\
81.1\end{array}$ & $\begin{array}{l}44.2 \\
18.8\end{array}$ & $\begin{array}{r}11.5 \\
1.8\end{array}$ & $\begin{array}{l}\text { o+ } \\
0\end{array}$ & $\begin{array}{l}\circ \\
\circ\end{array}$ & $\begin{array}{l}\circ \\
\circ\end{array}$ & $\begin{array}{l}\circ \\
\circ\end{array}$ & $\begin{array}{l}0 \\
\circ\end{array}$ \\
\hline untere Extr. & $\begin{array}{l}\text { Oberschenkel } \\
\text { Unterschenkel }\end{array}$ & $\begin{array}{l}56.8 \\
66.7\end{array}$ & $\begin{array}{l}29.6 \\
27.1\end{array}$ & $\begin{array}{r}\mathrm{II.2} \\
6.2\end{array}$ & $\begin{array}{l}0^{+} \\
0\end{array}$ & $\begin{array}{l}\circ \\
\circ\end{array}$ & $\begin{array}{l}\circ \\
\circ\end{array}$ & $\begin{array}{l}\circ \\
\circ\end{array}$ & $\begin{array}{l}\circ \\
\circ\end{array}$ \\
\hline
\end{tabular}




\section{Haarzahl.}

Die grösste Haarbalgzahl auf I qcm Hautflächeninhalt zeigt die Stirn des Falles 8 (1265), und die kleinste der Bauch und der Oberschenkel (Medialseite) des Falles ir (23) (s.. Tabelle 2).

Auch in der Durchschnittszahl der i Fälle nimmt die Stirn den grössten (846), und der Bauch den kleinsten (53) ein. Den zweitgrössten Wert zeigt der Scheitel (439), dann kommt der Oberarm (Ss) mit der Zahl 90, der Hals 86, der Rücken 83, der Oberarm (Ss) 8I usw. Ausser dem Bauch weist der Vorderarm (Bs) und der Oberschenkel (Ms) je kleinen Wert, d.h. 55 und 57 auf.

Bei den Körperteilen hat der Kopf wie auch bei den einzelnen Fällen weitaus grösste durchschnittliche Haarzahl; die Haardichtigkeit des Rumpfes und der Extremitäten ist weit niedriger als die des Kopfes, und zwar ist sie zwischen dem Rumpf und den Extremitäten fast gleichgradig in der Durchschnittszahl wie auch meist bei den einzelnen Fällen.

Beim Rumpf ist die Haarzahl an der dorsalen Seite grösser als an der ventralen im Durchschnitt, desgleichen auch bei den einzelnen Fällen.

Von den Extremitäten übertreffen die oberen Extremitäten in der Haardichtigkeit etwas die unteren, obwohl kein merklicher Unterschied zwischen Proximalis und Distalis zu erkennen ist.

Zwischen dem Ober- und Vorderarm besteht zwar kein merklicher Unterschied der Haardichtigkeit in der Durchschnittszahl, aber falls hier ein grosser Unterschied bei einzelnen Fällen vorhanden ist, übertrifft der Oberarm immer den Vorderarm. Die Streckseite der oberen Extremitäten übertrifft im allgemeinen deutlich die Beugeseite.

Es besteht kein Unterschied zwischen dem Ober- und Unterschenkel. Beim Oberschenkel weist die Lateralseite höhere Haardichtigkeit als die Medialseite auf, während beim Unterschenkel die Streck- und Beugeseite gleiche Haardichtigkeit besitzen.

\section{Zahl der Haargruppen.}

Wir konnten bei der Stirn die einzelnen Haargruppen nicht bestätigen, weil sich hier keine deutlichen haarfreien Zwischenräume wie bei anderen Körperteilen zwischen den einzelnen Haargruppen erkennen liesen. So haben wir, abgesehen von der Stirn, an 14 Körperteilen die Dichtigkeit der Haargruppen untersucht.

Den grössten Wert der Haargruppenzahl in I qcm Hautflächeninhalt zeigt der Scheitel des Falles I (187). Der kleinste betrug 23 am Bauch und Oberschenkel (Ms) des Falles I I und am Oberschenkel (Ms) des Falles 4. 
In der Durchschnittszahl der I I Fälle ist die Dichtigkeit der Haargruppen weitaus am grössten am Scheitel, sie betrug x 50 . Es liegt kein merklicher Unterschied zwischen anderen Körperteilen ausser dem Kopf vor, der Wert ergibt ca. ein Drittel der Zahl am Scheitel, und zwar zeigt der Bauch und Oberschenkel (Ms) den kleinsten Wert von je 35 und 36 .

Bei den Hauptkörperteilen ist die Haargruppenzahl am Kopf weitaus am grössten, der Rumpf und die Extremitäten zeigen miteinander ähnliche Haargruppendichtigkeit, nämlich ca. ein Drittel des Wertes am ersteren. Am Rumpf ist die Dorsalseite etwas dichter als die Ventralseite.

Es liegt bei den Extremitäten zwischen Proximalis und Distalis, oberen und unteren Extremitäten, Beugeseite und Streckseite der oberen Extremitäten, Ober- und Unterschenkel und Streck- und Beugeseite des Unterschenkels kein deutlicher Unterschied vor ; ein ziemlich grosser Unterschied besteht aber zwischen der Medial- und Lateralseite des Oberschenkels.

\section{Zahl der verschiedenen Haargruppenarten.}

a. Dichtigkeit der einzelnen Haargruppenarten in den verschiedenen Körperteilen.

Im Durchschnittswert von I I Chinesen ist die Zahl der I-haarigen Haargruppe in I qcm Hautflächeninhalt, abgesehen von der Stirn, weil sich hier die einzelnen Haargruppen nicht unterscheiden liessen, am grössten an der Beugeseite des Vorderarmes, dann folgen Oberarm (Bs), Unterschenkel (Ss), Oberschenkel (Ls), Unterschenkel (Bs) usw., und zwar ist sie nicht so verschieden zwischen allen untersuchten Körperteilen, nur weitaus am kleinsten am Scheitel.

Die Zahl der 2-haarigen Gruppe ist weitaus am reichlichsten am Scheitel, dann der Reihe nach am Rücken wie auch am Vorderarm (Ss), Gesäss, Brust wie auch Oberarm (Ss) ziemlich reichlich und sehr minder an anderen Körperteilen, besonders am mindesten an der Beugeseite des Vorderarmes, obgleich sie von I-haariger Gruppe die höchste Dichtigkeit zeigt.

Die 3-haarige Gruppe ist weitaus am reichlichsten am Scheitel, sehr minder an anderen Körperteilen, besonders am Bauch, an der Beugeseite des Ober- und Vorderarmes.

Die 4-haarige Gruppe findet sich fast lediglich am Scheitel, wenig am Hals und spurweise am Rücken, an der Streckseite des Oberarmes und an der Lateralseite des Oberschenkels.

Nur am Scheitel findet sich die 5-haarige Gruppe ziemlich reichlich, die 6-haarige mässig und die 7- und 9-haarige Gruppe spurweise.

Bei den Hauptkörperabschnitten ist die I-haarige Gruppe an den Extre- 
mitäten am reichlichsten, dann folgen der Rumpf und schliesslich der Kopf. Am Rumpf findet sich kein Unterschied zwischen der Dorsal- und Ventralseite. Die oberen Extremitäten übertreffen in der Dichtigkeit der I-haarigen Haargruppe die unteren. Zwischen Proximalis und Distalis der Extremitäten, dem Ober- und Unterschenkel ist kein Unterschied bemerkbar. Anderseits übertrifft die Streckseite der oberen Extremitäten die Beugeseite, der Unterschenkel der unteren Extremitäten den Oberschenkel.

Die Dichtigkeit der 2-haarigen Gruppe ist am Kopf weitaus am grössten, am Rumpf etwas grösser als an den Extremitäten. Sie ist am Rumpf an der Dorsal- als Ventralseite, von den oberen Extremitäten an der Streck- als Beugeseite und an den oberen als unteren Extremitäten je grösser; es liegt aber zwischen Proximalis und Distalis der Extremitäten, dem Ober- und Vorderarm und dem Ober- und Unterschenkel kein Unterschied vor.

Die 3-haarige Gruppe ist bei den Hauptkörperteilen am Kopf weitaus am reichlichsten vorhanden, dann sehr minder am Rumpf und Extremitäten in ähnlicher Dichtigkeit. Die Streckseite der oberen Extremitäten übertrifft die Beugeseite, gleich wie die Dorsalseite des Rumpfes die Ventralseite und der Oberschenkel den Unterschenkel, in der Dichtigkeit der 3-haarigen Gruppe. Zwischen den oberen und unteren Extremitäten, dem Proximalis und Distalis der Extremitäten und dem Ober- und Vorderarm ist kein Unterschied vorhanden.

Die 4-haarige oder über 4-haarige Gruppe kommen fast alle am Kopfe, sehr selten an anderen Körperteilen vor.

b. Frequenz der verschiedenen Haargruppenarten in den einzelnen Körperteilen.

Am Scheitel kommt die 2-haarige Gruppe am reichlichsten vor, dann folgt die 3 -haarige, die 4 -haarige ist relativ reichlich, während die 5 -haarige und über 5 -haarige relativ selten sind.

Am Hals nehmen die 1-, 2- und 3-haarige Gruppe ungefähr gleichen Prozentsatz ein, die 4-haarige kommt aber viel seltener als die obengenannten Gruppen vor.

An Brust, Bauch, Rücken und Gesäss ist der Prozentsatz der I- und 2haarigen Gruppe fast gleich, dagegen der der 3-haarigen viel kleiner.

An der Streckseite des Ober- und Vorderarmes ist der Prozentsatz der I-haarigen Gruppe fast gleich wie der der 2-haarigen; bei der 3-haarigen ist er viel kleiner als der der obengenannten Gruppen.

An der Beugeseite des Ober- und Vorderarmes ist der Prozentsatz der I-haarigen Gruppe besonders gross, der der 2-haarigen ist nicht mehr als ein Drittel des Prozentsatzes der I-haarigen und schliesslich ist die 3 -haarige 
Gruppe noch viel seltener als die 2-haarige.

Am Ober- und Unterschenkel kommt die I-haarige Gruppe sehr häufig vor, der Prozensatz der 2-haarigen Gruppe ist ungefähr die Hälfte des Prozentsatzes der I-haarigen; die 3-haarige kommt noch viel seltener als die 2haarige vor.

Bei den Hauptkörperabschnitten sind die Prozentsätze der verschiedenen Haargruppenarten am Kopf dieselben, wie oben am Scheitel erwähnt. An den Extremitäten ist im Gegensatz zum Kopfe die Häufigkeit der I-haarigen Gruppe weitaus am grössten, die 2-haarige Gruppe zeigt bedeutend kleineren Prozentsatz als den der I-haarigen, die 3-haarige Gruppe weist sehr kleinen Prozentsatz auf; die 4-haarige Gruppe ist sehr selten vorhanden.

Am Rumpf zeigen die Verhältnisse der verschiedenen Haargruppenarten das Verhalten zwischen dem Kopf und den Extremitäten, d.h. die Häufigkeit der I- und 2-haarigen Gruppe ist hier fast gleichmässig, die 3-haarige kommt viel seltener als die ersteren vor.

An der Dorsalseite des Rumpfes ist die Zusammensetzung der verschiedenen Haargruppenarten fast gleich wie an der Ventralseite, indem hier die I- und 2-haarige Gruppe am reichlichsten und der Prozentsatz der beiden Gruppen fast gleich ist. Die 3-haarige Gruppe ist viel seltener als die ersteren, und etwas häufiger an der Dorsal- als an der Ventralseite. An der Dorsalseite ist auch die 4-haarige Gruppe zu finden.

Zwischen den oberen und unteren Extremitäten ist fast kein Unterschied in der Zusammensetzung der verschiedenen Haargruppenarten zu erkennen. Es verhält sich so, dass die I-haarige Gruppe weitaus am reichlichsten, die 2-haarige in der Hälfte des Prozentsatzes der I-haarigen und die 3-haarige sehr selten vorkommen.

Zwischen dem Proximalis und Distalis des Ober- und Vorderarmes ist kein Unterschied bezüglich der Zusammensetzung der verschiedene Haargruppenarten zu finden, die Prozentsätze sind hier ganz gleich wie bei den oberen und unteren Extremitäten. Zwischen der Streck- und Beugeseite der oberen Extremitäten findet sich ein kleiner Unterschied, d.h. an der Streckseite ist der Prozentsatz der I-haarigen Gruppe fast gleich dem der 2-haarigen, die 3-haarige ist aber viel kleiner als die beiden ersteren. Anderseits ist an der Beugeseite die I-haarige Gruppe überwältigend häufig, die 2-haarige Gruppe kommt seltener als die I-haarige und die 3-haarige sehr selten vor.

Zwischen dem Ober- und Unterschenkel findet sich fast die gleiche Zusammensetzung der verschiedenen Haargruppenarten, d.h. die I-haarige Gruppe zeigt in der Häufigkeit die Hälfte der I-haarigen, noch seltener ist die 3-haarige Gruppe. Die Unterschiede zwischen der Häufigkeit der verschiedenen Haargruppenarten scheinen aber am Unterschenkel grösser als 
am Oberschenkel zu sein.

Aus Obenerwähnten kann man den Schluss ziehen, dass die Schwankung der Haardichtigkeit nach den verschiedenen Körperteilen der Haargruppendichtigkeit fast parallel geht und dass an den Körperteilen, wo das Haar dichter vorhanden ist, der Prozentsatz der Haargruppen, die aus mehreren Haaren bestehen, auch grösser ist.

\section{Rassenverhältnisse.}

\section{r. Vergleich mit Japanern.}

\section{a. Haarzahl.}

Nach Taniguchi und Shibayama liegt bei Japanern der Rumpf in der Haardichtigkeit in der Mitte zwischen dem Kopf und den Extremitäten, dagegen ist bei unseren Chinesen kein Unterschied zwischen dem Rumpf und den Extremitäten vorhanden.

Bei Japanern verhält sich die Reihenfolge der Haardichtigkeit an den verschiedenen Körperteilen im Durchschnitt wie folgt: Stirn, Scheitel, Gesäss, Vorderarm (Ss), Rücken, Oberschenkel (Ls), Oberarm (Ss), Oberschenkel (Ms), Unterschenkel (Ss), Oberarm (Bs), Brust, Vorderarm (Bs), Unterschenkel (Bs) und Bauch. Dagegen bei Chinesen: Stirn, Scheitel, Vorderarm (Ss), Hals, Rücken, Oberarm (Ss), Gesäss, Oberschenkel (Ls), Brust, Oberarm (Bs), Unterschenkel (Bs) und Bauch. Im grossen und ganzen stimmt die Reihenfolge bei beiden Rassen miteinander überein, dabei ist besonders bemerkenswert die grosse Dichtigkeit an der Streckseite des Vorderarmes; die ausserordentlich höhere Dichtigkeit am Gesäss ist bei Japanern, aber nicht bei Chinesen wahrzunehmen.

Obgleich bei Japanern zwischen den oberen und unteren Extremitäten kein Unterschied in der Haardichtigkeit besteht, ist das Haar der Chinesen an den oberen Extremitäten viel dichter als an den unteren. Bei Japanern ist das Haar dichter proximal als distal, aber bei Chinesen ist es proximal gleich wie distal. Während bei Japanern der Oberschenkel bzw. Oberarm höhere Haardichtigkeit als der Unterschenkel bzw. Vorderarm besitzt, besteht bei Chinesen kein solcher Unterschied.

In anderer Hinsicht stimmen die beiden Rassen in der Verteilung der Haare miteinander gut überein. 
b. Haargruppen.

Bei den Japanern ist nach Taniguchi und Shibayama die Haargruppendichtigkeit grösser proximal als distal, aber bei unseren Chinesen ist sie proximal wie distal gleich.

Bei den Japanern ist die Haargruppendichtigkeit bei ${ }_{3} 3$ unter ${ }_{17}$ Fällen an der Beugeseite des Oberarmes grösser als an der Streckseite, dagegen bei unseren Chinesen ist sie bei 7 von Io Fällen (in einem Fall wurde die Beugeseite des Oberarmes nicht bestätigt) an der Streckseite grösser als der Beugeseite.

Ausserdem sind die beiden Menschenrassen bezüglich der Haargruppendichtigkeit gut übereinstimmend.

c. Zahl der verschiedenen Haargruppenarten.

Am Scheitel ist die Häufigkeit der verschiedenen Haargruppenarten im Durchschnitt bei 19 Japanern von Taniguchi und Shibayama der Reihe nach wie folgt: Die 3-haarige Gruppe (37.7\%), 2-haarige (29.4\%), 4-haarige (18.7\%). s-haarige $10.9 \%$ ), I-haarige gleich wie die 6-haarige $(2.4 \%)$, und schliesslich die 9-haarige (0.03\%). Anderseits ist sie bei unseren Chinesen: 2-haarige (36.7\%), 3-haarige (27.5\%), 4-haarige (14.8\%), 5haarige $(9.3 \%)$, r-haarige $(7.4 \%)$, 6-haarige (4.1\%), schliesslich 7- und 9haarige (unter $\mathrm{I} \%$ ).

Also bezüglich der Häufigkeit der 2- und 3-haarigen Gruppe besteht hier eine ganz umgekehrte Beziehung zwischen Chinesen und Japanern.

An der Brust ist die Reihenfolge bei Japanern folgendermassen: 2haarige Gruppe $(47.3 \%)$, I-haarige $(46.3 \%)$, 3-haarige $(6.3 \%)$ und 4 -haarige (0.1\%); bei Chinesen: I-haarige $(48.9 \%)$, 2-haarige $(46.7 \%)$, 3-haarige $(6.7 \%)$. Hier kann man leicht ersehen, wieweit sich ähnlicher Zusammenhang bei beiden Rassen findet.

Am Bauch der Japaner treten verschiedene Haargruppenarten der Reihe nach in folgender Häufigkeit auf: I-haarige Gruppe (59.6\%), 2-haarige (39. $\mathrm{\%} \%$ ), 3-haarige $(\mathrm{x} .23 \%)$; dies verhält sich bei Chinesen wie folgt: Ihaarige Gruppe (51.4\%), 2-haarige (45.7\%), 3-haarige (2.9\%). Also ist hier der Prozentsatz der I-haarigen Gruppe bei Japanern, dagegen der 2- und 3-haarigen Gruppe bei Chinesen etwas grösser als bei anderer Rasse.

Am Rücken ist die Reihenfolge der Häufigkeit der verschiedenen Haargruppenarten im durchschnittlichen Prozentsatz bei Japanern folgendermassen: 2-haarige Gruppe (46.I\%), r-haarige (45.9\%), 3-haarige (7.9\%), 4-haarige (0.1\%) und 5-haarige (0.08\%). Anderseits ist sie bei Chinesen : 2-haarige Gruppe (49.0\%), I-haarige (38.8\%), 3-haarige (1 2.2\%). Bei beiden Rassen ist die Häufigkeit und deren Reihenfolge der verschiedenen Haar- 
gruppenarten fast gleich, aber nur bei Chinesen fehlt die 4- und s-haarige Gruppe.

Am Gesäss von Japanern ist die Reihenfolge wie folgt : I-haarige Gruppe $(4 \mathrm{r} .2 \%)$, 2 -haarige $(37.6 \%)$, 3-haarige $(20.79 \%)$ und 4 -haarige $(0.39 \%)$, dagegen bei Chinesen: I-haarige sowie 2-haarige Gruppe (je 46.0\%), 3-haarige $(8.0 \%)$. Also ist die Häufigkeit der 3 -haarigen Gruppe bei Japanern viel grösser als bei Chinesen, und die 4-haarige kommt nur bei ersteren vor.

An der Beugeseite des Oberarmes zeigt die Zusammensetzung der verschiedenen Haargruppenarten nach der Reihenfolge des durchschnittlichen Prozentsatzes bei Japanern folgendes : I-haarige Gruppe (49.9\%), 2-haarige (33.8\%) und schliesslich 3-haarige ( $16.3 \%)$; aber bei Chinesen: I-haarige Gruppe (76.8), 2-haarige (21.4\%) und 3-haarige (1.8\%). Also ist hier die I-haarige Gruppe viel häufiger bei den Chinesen als bei den Japanern, und bei Japanern die 2- und 3-haarige Gruppe viel reichlicher als bei Chinesen.

An der Streckseite des Vorderarmes bei Japanern: I-haarige Gruppe ( $56.6 \%)$, 2-haarige $(26.2 \%)$, 3-haarige ( $16.1 \%)$, 4-haarige (0.3\%) und 5 -haarige (0.1\%), dagegen bei Chinesen: 2-haarige (45.3\%), r-haarige (41.5\%), 3haarige ( $13.2 \%)$. Hier ist die Beziehung zwischen den beiden Rassen ziemlich verschieden. Obgleich bei Japanern der Prozentsatz zwischen der Ihaarigen und 2-haarigen Gruppe sehr verschieden ist, stimmt der Prozentsatz der beiden Haargruppenarten bei Chinesen überein. Die 4- und 5-haarige Gruppe erscheinen nur bei Japanern.

An der Lateralseite des Oberschenkels bei Japanern : I-haarige Gruppe ( $5 \mathrm{I} .3 \%)$, 2-haarige $(30.5 \%)$, 3-haarige $(18.2 \%)$; bei Chinesen: I-haarige (65.3\%), 2-haarige (24.5\%), 3-haarige (10.2\%). Also ist die 2- und 3-haarige Gruppe bei Japanern, dagegen die I-haarige bei Chinesen relativ häufiger.

An der Medialseite des Oberschenkels bei Japanem : I-haarige Gruppe $(50.0 \%)$, 2-haarige (36.1\%) und 3-haarige ( $13.9 \%)$. Übereinstimmend kommt bei beiden Rassen die 3-haarige Gruppe selten vor. Obgleich der Prozentsatz der I- und 2-haarigen Gruppe bei Japanern gleich ist, findet sich bei Chinesen die I-haarige Gruppe sehr viel häufiger als die 2-haarige.

An der Beugeseite des Unterschenkels bei Japanern : I-haarige Gruppe (50.3\%), 2-haarige (34.5\%), 3-haarige (14.9\%); dagegen bei Chinesen : I-haarige $(66.0 \%)$, 2 -haarige $(29.8 \%)$ und 3 -haarige $(4.2 \%)$. Also ist der Unterschied $z$ wischen den Prozentsätzen der verschiedenen Haargruppenarten bei Chinesen grösser als bei Japanern.

An der Streckseite des Unterschenkels bei Japanern : I-haarige Gruppe $(56.0 \%)$, 2-haarige $(30.0 \%)$, 3-haarige ( $13.4 \%)$ und 4-haarige (1.1 \%); dagegen bei Chinesen: I-haarige Gruppe $(68.8 \%)$, 2-haarige (25.0\%), 3haarige $(6.2 \%)$. Also ist die Verschiedenheit der Häufigkeit zwischen der 
I-, 2- und 3-haarigen Gruppe etwas grösser bei Chinesen als bei Japanern, die 4-haarige Gruppe war nur bei Japanern erkennbar.

Zusammenfassend kann man Obiges folgendermassen schildern: Die Japaner und Chinesen stimmen in der Zusammensetzung der verschiedenen Haargruppenarten an Brust und Rücken miteinander ganz gut überein. Die Verschiedenheit zwischen ihnen liegt in folgender Hinsicht: Am Scheitel kommt bei Japanern die 3-haarige Gruppe, dagegen bei Chinesen die 2haarige Gruppe häufiger, an der Beugeseite des Oberarmes und Unterschenkels und an der Lateralseite des Oberschenkels ist die 2- und 3-haarige Gruppe bei Japanern, die I-haarige Gruppe bei Chinesen häufiger als bei anderer Rasse, an der Medialseite des Oberschenkels ist bei Chinesen die I-haarige Gruppe weit mehr vorhanden als die 2-haarige, im Vergleich mit Japanern.

An den obenerwähnten Körperteilen kommen bei Chinesen wie auch bei Japaneren dieselben Haargruppenarten vor, aber in ihrer Zusammensetzung sind sie bei beiden Rassen voneinander etwas verschieden.

Im folgenden handelt es sich um die Körperteile, wo irgendeine Haargruppenart nur bei Japanern oder Chinesen zum Vorschein kommt : An der Streckseite des Vorderarmes und am Rücken kamen die 4- und 5-haarige Gruppe, am Gesäss und an der Streckseite des Unterschenkels kam die 4-haarige Gruppe nur bei Japanern vor.

Aus Obenerwähnten kann man sogleich erkennen, dass bei Japanetn die aus vielen Haaren bestehenden Haargruppenarten, die an denselben Körperteilen bei Chinesen nicht bestätigt wurden, an manchen Körperteilen aufgefunden wurden, und dass aber solche Körperteile, wo die aus vielen Haaren bestehenden Haargruppenarten, die bei Japanern an denselben Körperteilen nicht auffindbar waren, nur bei Chinesen bestätigt wurden, niemals vorhanden waren.

Schliesslich waren solche Körperteile, an welchen die Zusammensetzung verschiedener Haargruppenarten zwischen den Japanern und Chinesen sehr verschieden und sogar nur bei Japanern die aus vielen Haaren bestehenden Gruppen vortraten, das Gesäss und die Streckseite des Vorderarmes und Unterschenkels.

Also kann man hier den Schluss ziehen, dass die Chinesen die Haargruppen, die aus einem oder wenigen Haaren bestehen, reichlicher, dagegen die Haargruppenarten, die aus mehreren Haaren bestehen, weniger als die Japaner besitzen.

\section{Vergleich mit einigen Rassen.}

Wenn die Befunde an unserem chinesischen Material mit den bei einigen Rassen von Taniguchi und Shibayama verglichen werden, ergibt sich 
Die Dichtigkeit der Körperbehaarung bei den Chinesen.

Tabelle 14. Prozentsatz der verschiedenen Haargruppenarten.

\begin{tabular}{|c|c|c|c|c|c|c|c|c|c|c|}
\hline Körperteile & \multicolumn{10}{|c|}{ Hinterhaupt } \\
\hline Haargruppen & $\mathrm{I}-\mathrm{Hg}$ & $2-\mathrm{Hg}$ & $3-\mathrm{Hg}$ & $4-\mathrm{Hg}$ & $\mathrm{s}-\mathrm{Hg}$ & $6-\mathrm{Hg}$ & $7-\mathrm{Hg}$ & $8-\mathrm{Hg}$ & $9-\mathrm{Hg}$ & $\begin{array}{l}\text { Haargruppen- } \\
\mathrm{zahl} \text { in I qcm }\end{array}$ \\
\hline Bantu (M'gonie) & $1.2 \%$ & $18.8 \%$ & $30.6 \%$ & $24.7 \%$ & $23.5 \%$ & $2.4 \%$ & - & - & -- & 85 \\
\hline Bantu (Xosa) & - & 17.5 & 32.5 & 28.1 & 13.2 & $7 \cdot 9$ & 一 & 一 & - & 114 \\
\hline Deutsche & - & $x .5$ & 24.3 & 22.1 & 20.6 & 22.9 & $8.8 \%$ & - & 一 & 136 \\
\hline Finne & 一 & 19.5 & 25.8 & 21.5 & 18.4 & 14.0. & I.I & - & - & 93 \\
\hline Ainu & 1.5 & 13.2 & 25.5 & 22.1 & 20.6 & II. 8 & $4 \cdot 4$ & $1.5 \%$ & - & 67 \\
\hline Koreanerin & - & I 2.7 & 24.6 & 26.4 & 23.2 & 11.3 & 2.I & - & - & 142 \\
\hline Japaner & 14.7 & 22.9 & 22.9 & 15.6 & 20.2 & 6.4 & $0^{+}$ & $0+$ & o+ & 109 \\
\hline Chinese & 7.4 & 36.9 & 27.5 & 14.8 & $9 \cdot 3$ & 4.1 & $0^{+}$ & - & o+ & I5O \\
\hline
\end{tabular}

\begin{tabular}{|c|c|c|c|c|c|c|c|c|}
\hline \multicolumn{3}{|c|}{ Bauch } & \multicolumn{5}{|c|}{ Rücken } \\
\hline $\mathrm{I}-\mathrm{Hg}$ & $2-\mathrm{Hg}$ & $3-\mathrm{Hg}$ & $\begin{array}{c}\text { Haargruppen- } \\
\text { zahl in I qcm }\end{array}$ & $\mathrm{I}-\mathrm{Hg}$ & $2-\mathrm{Hg}$ & $3-\mathrm{Hg}$ & $4-\mathrm{Hg}$ & $\begin{array}{c}\text { Haargruppen- } \\
\text { zahl in I qcm }\end{array}$ \\
\hline $22.2 \%$ & $72.2 \%$ & - & 28 & $12.0 \%$ & $68.0 \%$ & $20.0 \%$ & - & 25 \\
45.8 & 33.3 & $20.5 \%$ & 24 & 27.1 & 41.9 & 27.1 & - & 31 \\
31.6 & 60.1 & 7.9 & 38 & 19.4 & 52.8 & 28.0 & - & 36 \\
67.7 & 19.4 & 12.9 & 31 & 29.0 & 61.8 & 8.8 & - & 34 \\
24.1 & 44.8 & 31.1 & 29 & 13.5 & 45.9 & 40.5 & - & 37 \\
47.8 & 39.1 & 13.0 & 23 & 31.7 & 56.1 & 12.2 & - & 41 \\
33.3 & 66.6 & - & 24 & 27.4 & 50.0 & 20.4 & $2.2 \%$ & 46 \\
51.4 & 54.7 & 2.9 & 35 & 38.8 & 49.0 & 12.2 & 0 & 49 \\
\hline
\end{tabular}

\begin{tabular}{|c|c|c|c|c|c|c|c|c|c|}
\hline \multicolumn{5}{|c|}{ Oberarm } & \multicolumn{5}{|c|}{ Vorderarm } \\
\hline $\mathrm{I}-\mathrm{Hg}$ & $2-\mathrm{Hg}$ & $3-\mathrm{Hg}$ & 4-Hg & $\begin{array}{l}\text { Haargruppen- } \\
\mathrm{zahl} \text { in I qcm }\end{array}$ & $\mathrm{I}-\mathrm{Hg}$ & $2-\mathrm{Hg}$ & $3-\mathrm{Hg}$ & 4-Hg & $\begin{array}{l}\text { Haargruppen- } \\
\mathrm{zahl} \text { in } \mathrm{I} \mathrm{qcm}\end{array}$ \\
\hline $17.9 \%$ & $60.7 \%$ & $21.4 \%$ & 一 & 28 & $20.0 \%$ & $68.0 \%$ & $8.0 \%$ & $4.0 \%$ & 25 \\
\hline $37 \cdot 1$ & 37.1 & 25.7 & - & 35 & 33.0 & 40.0 & 26.7 & 一 & 30 \\
\hline 24.0 & 36.6 & 39.0 & -- & $4 I$ & 6.1 & 54.5 & 36.4 & 3.1 & 33 \\
\hline 26.4 & 42.1 & 31.6 & - & 38 & 16.1 & 48.4 & 35.5 & - & $3 I$ \\
\hline 10.3 & 43.6 & $5 x .3$ & $2.2 \%$ & 42 & $17 \cdot x$ & 37.1 & $45 \cdot 7$ & 一 & 35 \\
\hline 23.0 & 59.0 & 18.0 & - & 6I & 21.6 & 64.7 & 13.7 & - & $5 \mathrm{I}$ \\
\hline 36.4 & 36.4 & 27.3 & 一 & 33 & 31.0 & 48.7 & 20.7 & 一 & 29 \\
\hline 62.9 & $3 \mathrm{I} .5$ & 5.6 & o & 54 & 64.7 & 31.4 & 7.8 & - & 51 \\
\hline
\end{tabular}

\begin{tabular}{|c|c|c|c|c|c|c|c|c|}
\hline \multicolumn{5}{|c|}{ Oberschenkel } & \multicolumn{4}{|c|}{ Unterschenkel } \\
\hline $\mathrm{I}-\mathrm{Hg}$ & $2-\mathrm{Hg}$ & $3-\mathrm{Hg}$ & $4-\mathrm{Hg}$ & $\begin{array}{l}\text { Haargruppen- } \\
\mathrm{zahl} \text { in I qcm }\end{array}$ & $\mathrm{I}-\mathrm{Hg}$ & 2-Hg & $3-\mathrm{Hg}$ & $\begin{array}{l}\text { Haargruppen- } \\
\text { zahl in I qcm }\end{array}$ \\
\hline $18.5 \%$ & $47.6 \%$ & $42.9 \%$ & $1.0 \%$ & $2 I$ & $0.6 \%$ & $44.4 \%$ & $50.0 \%$ & 18 \\
\hline 50.0 & 34.6 & 15.4 & . - & 26 & 72.0 & 24.0 & 4.0 & 25 \\
\hline 30.0 & 36.1 & 33.3 & - & 30 & $23 . x$ & 46.1 & 30.8 & 26 \\
\hline 36.1 & 46.7 & 16.7 & - & 30 & 53.8 & 26.9 & x 9.2 & 26 \\
\hline$x 5.4$ & 34.6 & 50.0 & - & 26 & 22.2 & 33.3 & $44 \cdot 4$ & 27 \\
\hline 23.4 & 27.8 & 46.8 & 2.1 & 47 & 40.7 & 37.1 & 22.2 & 27 \\
\hline $2 \mathrm{x} .2$ & 60.6 & I8.I & - & 33 & 29.4 & $44 \cdot I$ & 26.4 & 34 \\
\hline 56.8 & 29.6 & II.2 & of & 44 & 66.7 & 27.1 & 6.2 & 48 \\
\hline
\end{tabular}


folgendes :

\section{a. Haarzahl.}

An der Stirn zeigt die Haarzahl in I qcm Hautflächeninhalt bei den verschiedenen Rassen der Reihe nach das Folgende: Japaner 802, Koreanerin 771, Deutsche 715, Chinese 713, Finne 671, Ainu 582, Bantu (Xosa) 422 usw. Die Dichtigkeit bei Chinesen ist mittelmässig unter allen untersuchten Rassen und gleicht dem Deutschen.

Am Hinterhaupt (u.z. bei Japanern und Chinesen statt des Hinterhauptes den Scheitel untersucht) ist sie bei Chinesen ( $6_{3} 0$ ) am grössten, dann kommen Deutsche 626, Koreanerin 504, Bantu (Xosa) 365, und am kleinsten bei Japanern 352. Auch hier verhält es sich bei Chinesen ähnlich wie bei Deutschen, indem sie die höchste Dichtigkeit des Haares zeigen.

Am Bauch : Deutsche 65 am grössten, Ainu 64, Chinese 53, dann weit kleiner Finne 45, Japaner 44, Bantu (Xosa) 4I, Bantu (M'gonie) 32 am kleinsten.

Am Rücken : Japaner IOI am grössten, Ainu 91, Chinese 83, Deutsche 75, Koreanerin 73, Bantu (Xoxa) 63, Finne 61, Bantu (M'gonie) so am kleinsten.

Der Wert von Chinesen liegt zwischen Ainu und dem Deutschen. Die Dichtigkeit von Chinesen gehört zu der grösseren Gruppe.

Oberarm: Koreanerin II9 am grössten, dann Ainu 100, Deutsche 79, Finne 77, Chinese 76, Bantu (Xosa) 67, Japaner 63 und Bantu (M'gonie) so. Die Chinesen, welche einen mittelmässigen Wert zeigen, gleichen dem Deutschen und Finnen.

Am Vorderarm: Koreanerin 104 am grössten, dann Ainu 86, Deutsche 77, Chinese 72, Bantu (M'gonie) 62, Bantu (Xosa) 6r und Japaner 54 am kleinsten.

Also ist hier der Wert von Chinesen mittelmässig und gleicht, wie am Oberarm, dem des Deutschen und Finnen.

Am Oberschenkel : Koreanerin 97 am grössten, Japaner wie auch Chinese 66, Ainu 63, Deutsche 60, Finne 52, Bantu (M'gonie) 46 und Bantu (Xosa) $4 \mathrm{I}$ am kleinsten. Die Chinesen zeigen den mittelmässigen Wert, welcher mit dem von Japanern ganz übereinstimmt, ähnlich mit dem bei Ainu und Deutschen.

Am Unterschenkel: Chinese 75 am grössten, Japaner 67, Ainu 62, Deutsche 57, Koreanerin 48, Finne 45, Bantu (M'gonie) wie auch (Xoxa) 4I. Die Chinesen, den grössten Wert zeigend, sind am ähnlichsten den Japanern.

Aus Obenerwähnten ergibt sich die folgende Zusammenstellung: Die 
Haardichtigkeit von Chinesen stimmt an Stirn, Scheitel, Rücken, Oberarm, Vorderarm und Oberschenkel mit der vom Deutschen überein. Die Chinesen und der Deutsche sind also miteinander am ähnlichsten von allen untersuchten Rassen in der Haardichtigkeit. Die Haardichtigkeit von Chinesen ist ausser Unterschenkel an allen Körperteilen von der bei Japanern verschieden.

Schliesslich zeigten die Chinesen den grössten Wert besonders am Scheitel und Unterschenkel, an anderen Körperteilen den mittelmässigen Wert von allen untersuchten Rassen.

\section{b. Haargruppen.}

Die Dichtigkeit der Haargruppen ist am Hinterhaupt (bei Japanern und Chinesen am Scheitel) am grössten bei Chinesen I 50 , dann folgt Koreanerin I42, Deutsche I36, Bantu (Xosa) I14, Japaner I09, Finne 93, Bantu (M'gonie) 85 , und bei Ainu 67 am kleinsten. Die Chinesen sind hier am ähnlichsten der Koreanerin und dem Deutschen, indem sie die höchste Dichtigkeit besitzen.

Am Bauch verhält es sich in der Reihenfolge der Haargruppendichtigkeit folgendermassen: Deutsche 38, Chinese 35, Finne 3 I, Ainu 29, Bantu (Xosa) sowie Japaner 24, Bantu (M'gonie) I8 als kleinste.

Am Rücken: Chinese 49, Japaner 46, Koreanerin 4I, Ainu 37, Deutsche 36, Finne 34, Bantu (Xosa) 31, Bantu (M'gonie) 25.

Am Oberarm: Koreanerin 6r, Chinese 54, Ainu 42, Deutsche 41, Bantu (M'gonie) 28. Hier gleichen die Chinesen der Koreanerin, die die höchste Dichtigkeit besitzt.

Am Vorderarm: Koreanerin wie auch Chinese $5 x$ bei weitem grösser, Ainu 34, Deutsche 35, Finne 3r, Bantu (Xosa) 30, Japaner 29, Bantu (M'gonie) 25. Die Chinesen und die Koreanerin besitzen die höchste Dichtigkeit.

Am Oberschenkel: Koreanerin 47, Chinese 44, Deutsche 30, Bantu (M'gonie) 2I. Die Chinesen gleichen fast der Koreanerin, welche die höchste Dichtigkeit besitzt.

Am Unterschenkel: Chinese 48, Japaner 34, Koreanerin wie auch Ainu 27, Deutsche wie auch Finne 26, Bantu (M'gonie) 25. Die Chinesen besitzen hier die bei weitem höchste Haargruppendichtigkeit.

Aus Obigen ergibt sich folgender Schluss : Die Chinesen besitzen an Hinterhaupt, Rücken, Vorderarm, Unterschenkel die höchste Haargruppendichtigkeit und an Bauch, Oberarm und Unterschenkel die zweitgrösste von allen untersuchten Rassen. Sie besitzen also mehr dichtere Haargruppen. Die Dichtigkeit der Haargruppen der Chinesen stimmt an Hinterhaupt, Oberarm, Vorderarm und Oberschenkel mit der der Koreanerin sehr gut überein, und am Rücken und Unterschenkel ähnelt die Dichtigkeit der der 
Japaner. Die Chinesen sind hier mit dem Deutschen mit Ausnahme des Unterschenkels an allen Körperteilen verschieden.

c. Zahl der verschiedenen Haargruppenarten.

Hinterhaupt (bei Japanern und Chinesen-Scheitel).

I-haarige Gruppe : Im Prozentsatz der I-haarigen Gruppe am Hinterhaupt zeigt der Japaner den höchsten Wert 14.7\%, dann Chinese 7.4\%, Ainu I. $5 \%$, Bantu (M'gonie) 1.2\%. Die I-haarige Gruppe fehlt bei Bantu (Xosa), Deutschen und Finnen.

2-haarige Gruppe. Chinese 36.9\%, Japaner 22.9\%, Finne $19.5 \%$, Bantu (M'gonie) $18.8 \%$, Deutsche $1.5 \%$.

3-haarige Gruppe: Bantu (Xosa) 32.5\%, Bantu (M'gonie) 30.6\%, Chinese $27.5 \%$, Finne $25.8 \%$, Koreanerin $24.6 \%$, Japaner $22.9 \%$ am kleinsten.

4-haarige Gruppe: Bantu (Xosa) 28.1\%, Koreanerin 26.4\%, Bantu (M'gonie) 24.7\%, Deutsche 22.1\%, Japaner I $5.6 \%$, Chinese $14.8 \%$.

5-haarige Gruppe : Bantu (M'gonie) 23.5\%, Koreanerin 23.2\%, Deutsche wie auch Ainu 20.6\%. Japaner 20.2\%, Finne $18.4 \%$, Bantu (Xosa) $13.2 \%$, Chinese $9.3 \%$.

6-haarige Gruppe: Deutsche 22.9\%, Finne 14.0\%, Ainu 1 1. $8 \%$, Koreanerin II.3\%, Japaner 6.4\%, Chinese 4. I\%, Bantu (M'gonie) $2.4 \%$ am kleinsten.

7-haarige Gruppe: Deutsche 8.8\%, Ainu 4.4\%, Koreanerin 2.1\%, Finne 1. $1 \%$; sie fehlt beim Bantu (Xosa und M'gonie), ist bei Chinesen und Japanern unter $\mathrm{I} \%$ vorzufinden.

8-haarige Gruppe war bei Ainu und Japanern, 9-haarige bei Chinesen und Japanern sehr selten nachzuweisen.

Aus dem Obigen ist ersichtlich, dass die I-haarige Gruppe bei Japanern am häufigsten, dann bei Chinesen reichlich, die 2-haarige bei Chinesen am häufigsten, dann bei Japanern reichlich vorhanden ist. Die 3-haarige war keinen merklichen Unterschieden zwischen den verschiedenen Rassen unterworfen, aber darunter waren die Chinesen und Japaner miteinander am ähnlichsten. Die 4-haarige war bei Japanern am seltensten, dann bei Chinesen selten vorhanden. Die s-haarige war bei Chinesen am wenigsten, aber am ähnlichsten zwischen Chinesen und Bantu (Xosa). Im Prozentsatz der 6-haarigen Gruppe sind die Chinesen den Japanern am ähnlichsten. Die 7-haarige Gruppe findet sich nicht bei Japanern und Bantu, dagegen bei Chinesen unter $\mathrm{r} \%$.

Am Hinterhaupt fehlt also die I-haarige Gruppe beim Deutschen und 
Finnen, aber bei ihnen war der Prozentsatz der 6- und 7-haarigen Gruppe mehr grösser als bei anderen Rassen. Es verhält sich bei Japanern und Chinesen umgekehrt. In den Prozentsätzen der 2-, 3- und 4-haarigen Gruppe, die am Scheitel am allgemeinsten vorhanden sind, stimmen die Chinesen mit den Japanern ganz gut überein. Hier sind die Chinesen am verschiedensten von Ainu.

Bauch.

I-haarige Gruppe: Den grössten Prozentsatz der I-haarigen Gruppe zeigt hier Finne $67.7 \%$, dann Chinese $51.4 \%$, Koreanerin $47.8 \%$, Bantu (Xosa) $45.8 \%$, Japaner $33.3 \%$, Deutsche $31.6 \%$, Ainu $24.1 \%$, und den kleinsten der Bantu (M'gonie) 22.3\%.

2-haarige Gruppe: Bantu (M'gonie) 72.2\%, Japaner 66.6\%, Deutsche $60.1 \%$, Chinese $45.7 \%$, Ainu $44.8 \%$, Koreanerin $39.1 \%$, Bantu (Xosa) $33.3 \%$, Finne $19.4 \%$.

3-haarige Gruppe; Ainu 31. $1 \%$, Bantu (Xosa) $20.5 \%$, Finne $12.9 \%$, Koreanerin $13.0 \%$, Chinese $2.9 \%$; sie fehlt beim Bantu (M'gonie) wie auch bei den Japanern.

Hier sind die Chinesen im Prozentsatz der I-haarigen Gruppe der Koreanerin, in dem der 2-haarigen den Ainu und in dem der 3-haarigen dem Bantu (M'gonie) wie auch den Japanern am ähnlichsten; im Prozentsatz der 2haarigen dem Deutschen und der Koreanerin wie auch in dem der 3-haarigen der Koreanerin am zweitähnlichsten.

Als Ganzes waren die Chinesen in der Zusammensetzung der verschiedenen Haargruppenarten der Koreanerin am ähnlichsten, dagegen von den Ainu am verschiedensten.

\section{Rücken.}

I-haarige Gruppe: Die Chinesen zeigen den höchsten Prozentsatz $38.8 \%$, dann Koreanerin 31.7\%, Finne 29.0\%, Bantu (Xosa) $27.1 \%$, Ainu 13.5\%, Bantu (M'gonie) $12.0 \%$.

2-haarige Gruppe: Bantu (M'gonie) 68.0\%, Finne 61.0\%, Koreanerin $56.1 \%$, Deutsche $52.8 \%$, Japaner $50.0 \%$, Chinese $41.9 \%$.

3-haarige Gruppe : Ainu $40.5 \%$, Deutsche $28.0 \%$, Bantu (Xosa) $27.1 \%$, Japaner $20.4 \%$, Koreanerin sowie Chinese $12.2 \%$, Finne $8.8 \%$.

4-haarige Gruppe: $\mathbf{2 . 2} \%$, Chinese unter $1 \%$. Sie ist bei anderen Rassen nicht wahrnehmbar.

Die Befunde also zeigen, dass im Rücken die Chinesen in der Dichtigkeit der I-haarigen Gruppe der Koreanerin am ähnlichsten sind und den höchsten Prozentsatz zeigen. Die Japaner sind hier am ähnlichsten dem Deutschen. 
Die Chinesen stimmen im Prozentsatz der 2-haarigen Gruppe mit den Japanern und Bantu (Xosa) und in dem der 3 -haarigen mit der Koreanerin vollständig überein.

Wie in der Tabelle ersichtlich, sind die Chinesen in der Zusammensetzung verschiedener Haargruppenarten der Koreanerin am ähnlichsten, und von Bantu (M'gonie) am verschiedensten.

\section{Oberarm.}

I-haarige Gruppe : Chinese $62.9 \%$, dann Japaner $36.4 \%$, Finne $26.4 \%$, Deutsche $24.0 \%$, Koreanerin $23.0 \%$, Bantu (M'gonie) $17.9 \%$, Ainu $10.3 \%$. 2-haarige Gruppe: Bantu (M'gonie) 60.7\%, Koreanerin $59.0 \%$, Ainu 43.6\%, Finne 42. $1 \%$, Bantu (Xosa) $37.6 \%$, Deutsche $36.6 \%$, Japaner $36.4 \%$, Chinese $31.5 \%$ am kleinsten.

3-haarige Gruppe: Ainu 51.3\%, Deutsche 39.0\%, Japaner 27.3\%, Bantu (M'gonie) wie auch Koreanerin $18.0 \%$, Chinese $5.6 \%$.

4-haarige Gruppe : Ainu 2.2\% am grössten, Chinese unter I \%, dagegen war sie bei anderen Rassen nicht zu bestätigen. Also zeigt hier die r-haarige Gruppe bei Chinesen den grössten und bei Japanern den zweitgrössten Prozentsatz, der aber nur die Hälfte des Prozentsatzes der Chinesen ausmacht. Der Prozentsatz der 2-haarigen Gruppe war bei Chinesen am kleinsten. Die 4-haarige Gruppe liess sich hier bei Ainu und Chinesen im minimalen Prosentsatz wahrnehmen.

Wie in der Tabelle 14 ersichtlich, besitzen hier die Chinesen die I-haarige Gruppe weitaus reichlicher, sie sind in der Zusammensetzung verschiedener Haargruppenarten sehr verschieden von anderen Rassen, darunter aber den Japanern am ähnlichsten.

\section{Vorderarm.}

I-haarige Gruppe: Chinese $64.7 \%$, Bantu (Xosa) $33.0 \%$, Japaner $31.0 \%$, Koreanerin $21.6 \%$, Bantu (M'gonie) $20.0 \%$, Deutsche 6.1\%.

2-haarige Gruppe : Bantu (M'gonie) 68.0\%, Koreanerin 64.7\%, Deutsche $54.0 \%$, Finne $48.4 \%$, Japaner $48.3 \%$, Bantu (Xosa) $40.0 \%$, Ainu $37.1 \%$, Chinese $31.4 \%$.

3-haarige Gruppe: Ainu 45.5\%, Finne 35.3\%, Deutsche $35.4 \%$, Japaner 20.7\%, Bantu (M'gonie) $8.0 \%$, Chinese $7.8 \%$.

4-haarige Gruppe : Bantu (M'gonie) $4.0 \%$, Deutsche $3.1 \%$; sie ist bei anderen Rassen nicht $\mathrm{zu}$ finden.

Die Chinesen sind also in der Zusammensetzung der verschiedenen Haargruppenarten am Vorderarm von anderen Rassen sehr verschieden. 
Oberschenkel.

I-haarige Gruppe: Chinese $56.8 \%$, Bantu (Xosa) $50.0 \%$, Finne $36.1 \%$, Deutsche $30.0 \%$, Japaner 21.2\%, Bantu (M'gonie) $18.5 \%$.

2-haarige Gruppe: Japaner 60.6\%, Deutsche $36.1 \%$. Chinese 29.6\%.

3-haarige Gruppe: Ainu 50.0\%, Koreanerin 46.8\%, Bantu (M'gonie) 42.9\%, Deutsche $36.1 \%$, Japaner $18.1 \%$, Bantu (Xosa) $15.4 \%$, Chinese II. $2 \%$.

4-haarige Gruppe: Koreanerin 2.1\%, Chinese unter $1 \%$; sie ist bei anderen Rassen nicht wahrzunehmen.

Die Befunde zeigen, dass am Oberschenkel der Prozentsatz der $\mathrm{x}$-haarigen Gruppe bei den Chinesen am grössten ist, und dass die Chinesen und der Bantu (Xosa) am ähnlichsten sind. Der Prozentsatz der 2-haarigen Gruppe ist bei Japanern am grössten und bei Chinesen am kleinsten, der der 3-haarigen bei Chinesen am kleinsten und beim Bantu (Xosa) am ähnlichsten, und mit Japanern am zweitähnlichsten. Die 4-haarige Gruppe ist nur bei Koreanerin und Chinesen in minimalem Prozentsatz vorhanden.

Schliesslich stimmt die Zusammensetzung der verschiedenen Haargruppenarten am Oberschenkel bei Chinesen, wie in der Tabelle $14 \mathrm{zu}$ ersehen ist, mit der des Bantu (Xosa) ganz gut überein und ist von der des Bantu (M'gonie) am verschiedensten.

Am Unterschenkel.

I-haarige Gruppe: Bantu (Xosa) 72.0\%, Chinese $66.7 \%$, Koreanerin 40.7\%, Japaner 29.4\%, Bantu (M'gonie) $0.6 \%$.

2-haarige Gruppe: Bantu (M'gonie) $44.4 \%$, Japaner sowie Deutsche $46.1 \%$, Chinese $27.1 \%$, Bantu (Xosa) $4.0 \%$.

3-haarige Gruppe: Bantu (M'gonie) 50.0\%, Ainu 44.4\%, Deutsche $30.8 \%$, Japaner $26.4 \%$, Chinese $6.2 \%$; sie ist beim Bantu (Xosa) $4.0 \%$ am kleinsten.

Am Unterschenkel ist der Prozentsatz der I-haarigen Gruppe bei Chinesen am ähnlichsten dem beim höchsten Prozentsatz zeigenden Bantu (Xosa). Der Prozentsatz der 2-haarigen Gruppe stimmt zwischen Finne, Bantu (Xosa) und Chinesen ganz gut überein. Im Prozentsatz der 3-haarigen Gruppe sind die Chinesen dem den kleinsten Prozentsatz zeigenden Bantu (Xosa) am ähnlichsten.

Wie es auch in der Tabelle leicht ersichtlich ist, stimmt die Zusammensetzung verschiedener Haargruppenarten bei Chinesen am Unterschenkel, wie auch am Oberschenkel, mit der beim Bantu (Xosa) sehr gut überein, und ist der beim Finnen ziemlich gut ähnlich.

Fasst man das Obige zusammen, so scheinen die Chinesen in der Zusam- 
mensetzung der verschiedenen Haargruppenarten an den verschiedenen Körperteilen den Japanern am ähnlichsten, der Koreanerin am zweitähnlichsten und von dem Deutschen und Finnen am verschiedensten zu sein.

\section{Schluss.}

Unsere Hauptergebnisse über die Haare bei Chinesen wurden folgendermassen zusammengefasst.

x. Die Schwankungen der Haardichtigkeit nach den verschiedenen Körperteilen gehen mit den der Haargruppendichtigkeit im grossen und ganzen parallel.

2. Die Haargruppe, die aus vielen Haaren besteht, d.h. die 5- oder über s-haarige Gruppe, liegt meist an den Körperteilen, die relativ grosse Haardichtigkeit zeigen.

3. Zwischen den Japanern und Chinesen war die Haardichtigkeit an den meisten Körperteilen ziemlich verschieden, obwohl die Haargruppendichtigkeit sehr gut übereinstimmte. In der Zusammensetzung der verschiedenen Haargruppenarten besitzen die Japaner an manchen Körperteilen die Haargruppenarten, die aus relativ vielen Haaren bestehen, mehr als die Chinesen.

4. Im Vergleich der Chinesen mit einigen Rassen zeigen die Chinesen am Scheitel und Unterschenkel höhere Haardichtigkeit, sie stimmen in der Haardichtigkeit mit dem Deutschen sehr gut überein und sind von den Japanern und der Koreanerin sehr verschieden, aber im Gegensatz dazu stimmen sie in der Haargruppendichtigkeit mit den Japanern und der Koreanerin sehr gut überein, vom Deutschen vielmehr verschieden. In der Zusammensetzung der verschiedenen Haargruppenarten sind die Chinesen meistens den Japanern und der Koreanerin am ähnlichsten von allen untersuchten Rassen und von dem Deutschen, Finnen und Ainu sehr verschieden. 


\section{Literatur.}

I) Birker, F., Haut und Haare bei 6 Chinesenköpfen. Arch. f. Anthropol. Bd. 3. 1906.

2) Brunn, A., Haut. Handbuch der Anatomie v. Bardeleben. 1897. Jena.

3) Frederic, J., Untersuchungen über die Rassenunterschiede der menschlichen Kopf haare. Zeitschr. f. Morph. u. Anthropol. Bd. 9. 1906.

4) Jadassohn, J., Handbuch der Haut- und Geschlechtskrankheiten. Bd. I/r. Anatomie der Haut. 1927. Berlin.

5) Kato, S., Untersuchungen über die Haar- und Haargruppendichtigkeit bei den japanischen Feten. Folia anat. jap. Bd. 14. 1936.

6) Kurita, Y., Die Dichtigkeit der Körperbehaarung bei den japanischen Zwillingsfeten. Folia anat. jap. Bd. 17.1938.

7) Meyer-Lierheim, F., Die Dichtigkeit der Behaarung beim Fetus des Menschen und der Affen. Zeitschr. f. Morph. und Anthropol. Bd. 13. 1911.

8) Mogi, E., Untersuchungen über die Haardichtigkeit bei den Feten einiger Säugetierarten. Folia anat. jap. Bd. I . 1937.

9) Nehse, E., Beiträge zur Morphologie, Variabilität und Vererbung der menschlichen Kopfbehaarung. Zeitschr. f. Morph. und Anthropol. Bd. 36. 1936.

ro) Soumiern, Y. K., Studien über die Dichte der Körperbehaarung und Haargruppierung bei den Finnen. Acad. Sci. Finn. Scr. 25. 1925. (ref. im Anatomischen Bericht. Bd. 7).

II) Taniguchi, T., und Shibay ama, H., Die Dichtigkeit der Körperbehaarung bei einigen Rassen. Folia anat. jap. Bd. 13. 1935.

12) Taniguchi, T., und Shibayama, H., Die Dichtigkeit der Körperbehaarung bei den Japanern. Folia anat. jap. Bd. 13. 1935. 